\title{
Research Paper \\ Perception of femininity in different countries: a qualitative metasynthesis
}

Asie Omidvar Tehrani ${ }^{1}$, Fariba Zarani ${ }^{2}$, Shabnam Nohesara ${ }^{3}$, leili panaghi ${ }^{4}$, Mona Malekzadeh Moghani $^{5}$

1. Ph.D Candidate in Clinical Psychology, Faculty of Education \& Psychology, Shahid Beheshti University, Tehran, Iran.

2. Assistant Professor, Department of Psychology, Faculty of Education \& Psychology, Shahid Beheshti University, Tehran, Iran.

3. Associate Professor, Department of Psychiatry, School of Medicine, Iran University of Medical Science, Tehran, Iran.

4. Associate Professor, Department of Psychology, Family Research Institute, Shahid Beheshti University, Tehran. Iran.

5. Associate Professor, Department of Radiotherapy, School of Medicine, Shahid Beheshti University of Medical Sciences, Tehran, Iran.

Citation: Omidvar Tehrani A, Zarani F, Nohesara Sh, Panaghi L, Malekzadeh Moghani M. Perception of femininity in different countries: a qualitative metasynthesis. J of Psychological Science. 2021; 20(103): 1015-1038.

URL: https://psychologicalscience.ir/article-1-1168-fa.html
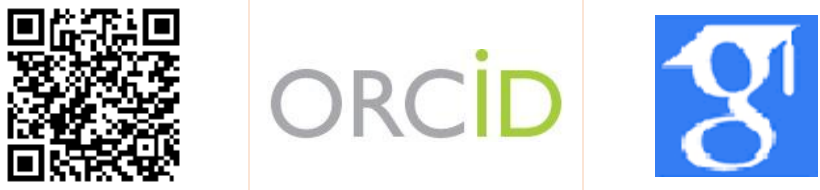

$\underline{10.52547 / J P S .20 .103 .1015}$

\section{A R T I C L E I N F O A B S T R A C T}

Keywords:

Femininity,

Perception of femininity,

Motherhood,

Culture,

Sexual agency

Received: 28 Feb 2021

Accepted: 26 Mar 2021

Available: 23 Sep 2021
Background: Femininity is a complicated term because it does not define a single phenomenon. Different perceptions of femininity are very dependent on one's background and knowledge of everyday actions that the meaning of femininity understood by them. In addition, the concept of femininity is close to the concept of gender, which in all societies is one of the basic structures of human identity and experiences, and the importance and role of it varies from society to society.

Aims: Since the definition of femininity varies depending on the culture and standards of each country, examining the perception of women in different countries can clarify various aspects of femininity.

Methods: In the present study, using the qualitative metasynthetic method of Noblit and Hare (1988), qualitative studies related to the subject of research in three databases Scopus, Sciencedirect and PubMed in the period 2000 to 2020 were examined. The search retrieved 377 articles and based on PRISMA method and inclusion and exclusion criteria, 19 articles selected to enter the research .

Results: Six different factors were found in the perception of femininity: sexual agency, motherhood, appearance and body, caregiver or self-care, independence and respect, and cultural factor.

Conclusion: Identifying different aspects of femininity can help researchers to explain and solve various problems and concerns of women.

* Corresponding Author: Fariba Zarani, Assistant Professor, Department of Psychology, Faculty of Education \& Psychology, Shahid Beheshti University, Tehran, Iran.

E-mail: f_zarani@sbu.ac.ir

Tel: (+98) 9123241637

2476-5740/ (C) 2021 The Authors. This is an open access article under the CC BY-NC-ND license

(https://creativecommons.org/licenses/by-nc/4.0/). 


\section{Extended Abstract}

\section{Introduction}

Male/Female Dichotomy is one of the most primitive human dualities, which is based on biological differences and is related to the field of gender. What goes beyond the two concepts of female and male is the definition of femininity and masculinity. Masculinity and femininity are gender-related cultural structures that apply to both individuals and groups (Moreno-Bella, Willis \& Moya, 2019).

Gender (as opposed to biologically dependent gender) is recognized as a category of femininity and masculinity that has a social structure and organizes behavior (Zajdo, 2007). Kanel has conceptualized gender as a set of social and fluid hierarchical relationships, which are used by subjects to relate to each other (Salazar, Guicolia, \& Ohman, 2016). Gender in all societies is one of the basic structures that shape the identity and experiences of individuals; however, its importance and role varies from one society to another and from one culture to another. Gender is one of the first social classifications that children learn in their societies today, and therefore the knowledge of gender stereotypes is evident from early childhood. In adulthood, people identify themselves with gender stereotypes that have been internalized during childhood (Kachel, Stephens, \& Needlech, 2016). These gender expectations are met through various social institutions such as the media, law and school. Gender socialization also occurs interpersonal, where family members, friends, and peers teach the child appropriate gender behavior. This process is known as the social construction of gender and gives meaning to words such as girl/boy, woman/man, and feminine/masculine (Zayn, Hindagno Sotlo, \& Messner, 2011). In her research, Nichols (2019) states that for women, 'doing' gender means successfully 'doing' femininity, which means that women consider traits, dispositions and behaviors that comprise appropriate femininity.

Although the term femininity contains a set of meanings, it generally refers to traits, behaviors, interests, origins, appearances, patterns, and expectations that are associated with being a woman during the process of socialization (Shee et al., 2014).
The concept of female identity (femininity) is also derived from social interactions with others. The woman compares herself to social norms, including common gender roles (Komatsu et al., 2014). Like beauty, femininity is often in the eyes of the viewer, too (Guillen Faby and Wilt, 2019). For example, in the study conducted by Pereira et al. (2019), men find great attraction to the characteristics of women's body and their behavior such as feminine face, dancing and voice. However, in the Hardy's study (2014), there was a weak or at best moderate correlation between sound quality and femininity perception. At the same time, certain characteristics are attributed to the ideal feminine face. In a study on a Chinese population, Han et al. (2020) stated that a woman's sexual characteristics are a sign of her health and fertility benefits, which are traits preferred by men. Other studies show that health, fertility and parenting abilities indicate women's femininity (Pereira et al., 2019). Mahalik et al. (2005) define femininity as the emotional, cognitive, and behavioral subordination of women to a set of social norms that have been passed down from generation to generation through culture. Because these social norms vary among different nations, races, social classes, and ethnicities, femininity is defined differently in each social group. In the field of cognitive manufacturing, there are value-cultural systems that determine the importance and relevance or the marginality of a subject. Also, due to the dominance of patriarchal culture, femininity has received less attention among various theories. On the other hand, in the field of women's studies, as part of the humanities, less attention has been paid to the study of their tendencies, plans, experiences, and feelings. However, effective programs for their mental health cannot be developed unless they have a precise and accurate knowledge of what is in a woman's mind and psyche about her feminine parts. Femininity as a scientific concept can have different meanings with countless interpretations. But when femininity is investigated as a whole, individual statements of femininity indicate different patterns. Given that so far no research has systematically investigated the concept of femininity, this study asks: what are the components of femininity from the perspective of 
women in different countries? Through answering this question, this study intends to review the different meanings of femininity in different countries and therefore to address the various parts of this broad concept from the perspective of women.

\section{Method}

In the present meta-analysis study, which was carried out systematically, Noblit and Harr's (1988) qualitative meta-ethnography approach was used in several stages. In the first stage, the title of the research, i.e. "The Perception of Femininity in Different Countries" was considered. In the second stage, only the studies qualified for entering the metaanalysis were included in the study. For this purpose, qualitative studies on the perception of femininity were searched in three databases: Scopus, Sciencedirect and PubMed, from 2000 to 2020, using a combination of the following keywords: femininity, feminine identity, being woman, perceived femininity.

At this stage, the inclusion criteria were: articles that examined femininity in women, directly measured women's experience of femininity, talked about the formation and criteria of femininity in a particular country, were published from 2000 to 2020, and were written in English. Exclusion criteria also included the articles examining femininity in various physical illnesses or specific psychological disorders, femininity in adolescents and men, femininity in literary, social, and historical works, as well as the articles whose full text was not available.

A total of 381 articles were retrieved based on the searches in the databases. After removing duplicate articles (8), 373 articles entered the initial screening. 347 articles were removed from the review process based on inclusion and exclusion criteria, and 26 ones were selected for full-text review. At this stage, 7 articles were removed from the research process because they were not in English or their full text was not available, and finally 19 articles were included in the final analysis (Figure 1).

Next, each of the selected studies was read carefully, with a focus on details in order to identify their key concepts and themes. In the fourth stage, the extracted key concepts were put together and the relationship between them was examined and considered. The fifth stage was to compare the key concepts extracted from each study with the key concepts of other studies, and finally in the last stage, the final result of meta-analysis (i.e. the factors involved in women's perception of femininity) was presented in six different aspects.

Figure 1: the process of reviewing and selecting articles (decision tree).

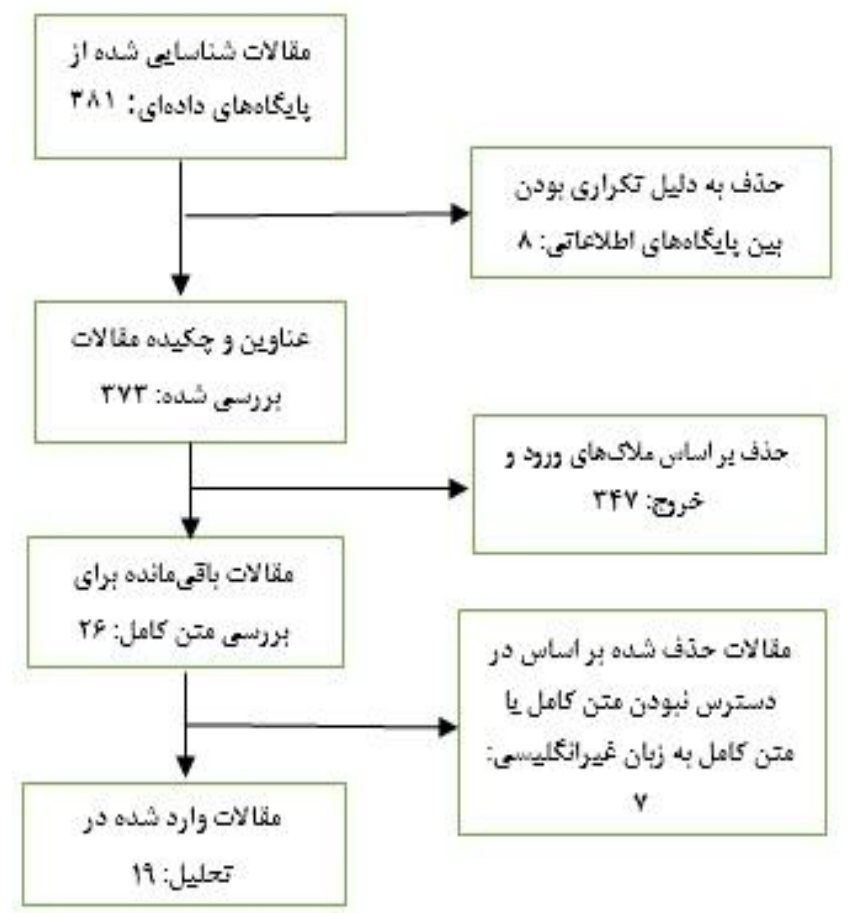

Results

The information extracted from the selected articles and their findings are given in the table below. The factors discussed in these articles were classified into six main areas: sexuality, motherhood, appearance and body, caregiver or self-care, independence and respect, and cultural factor, which will be discussed below. 
Table 1. Information extracted from the articles reviewed in the meta-analysis

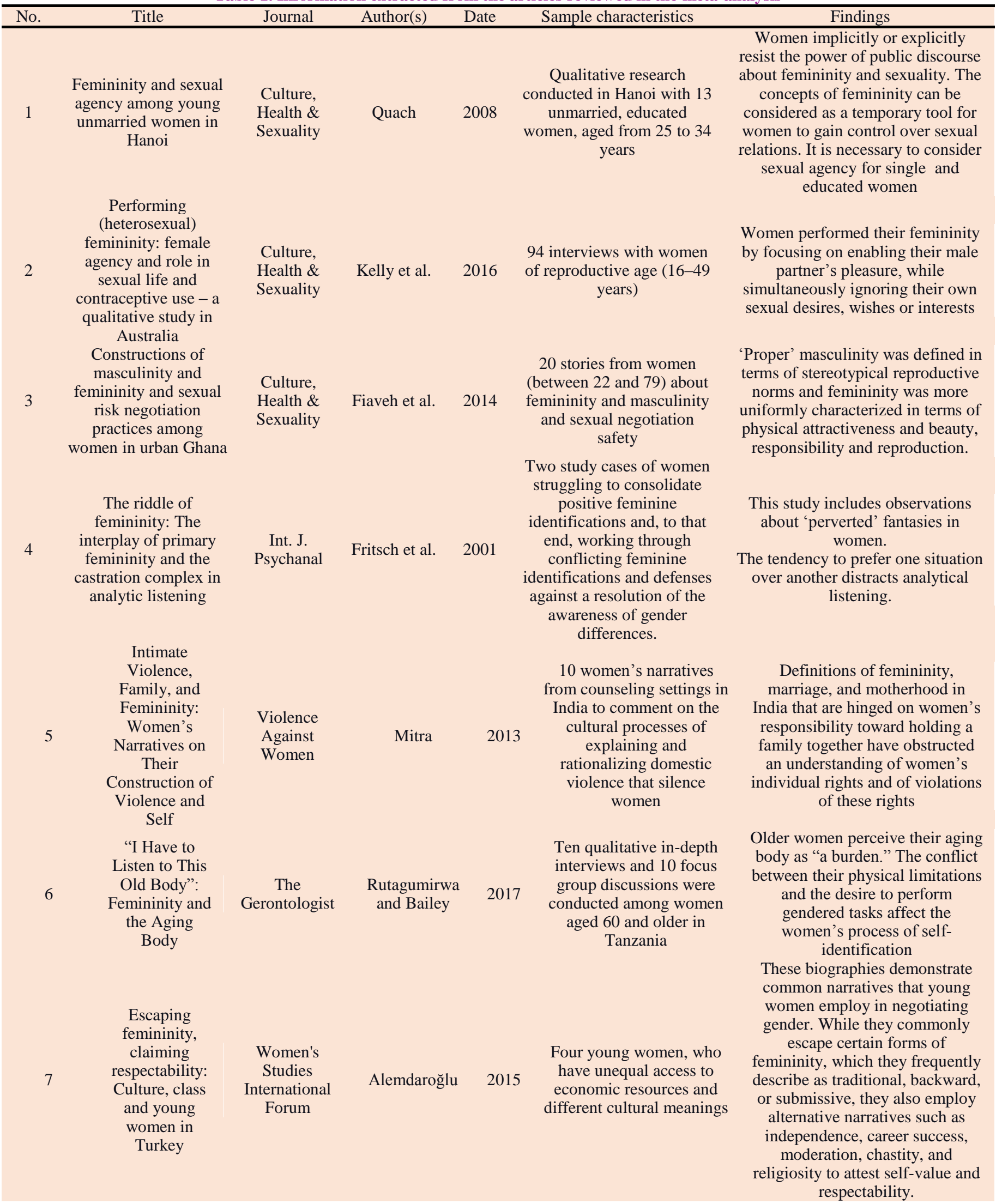




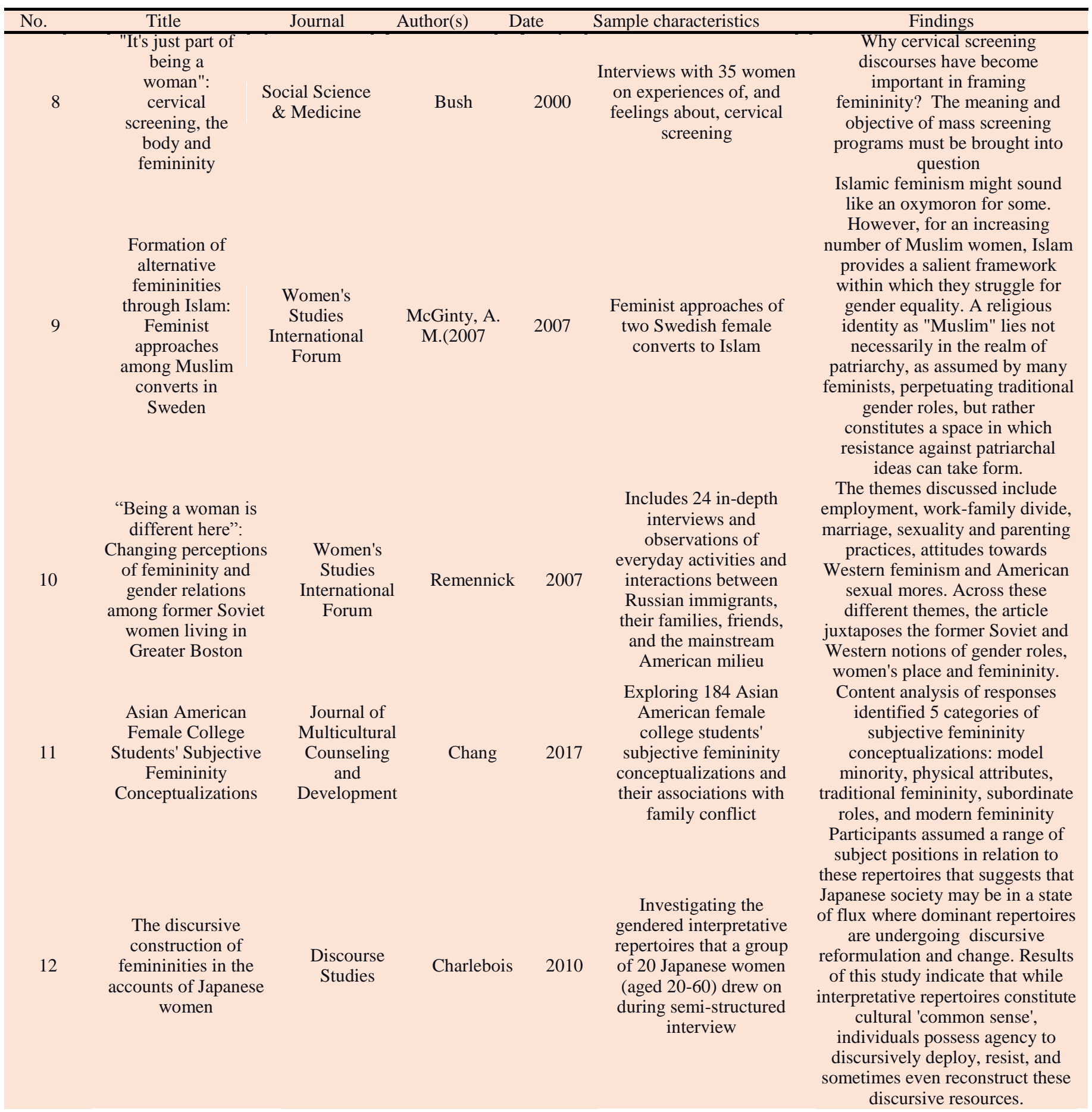




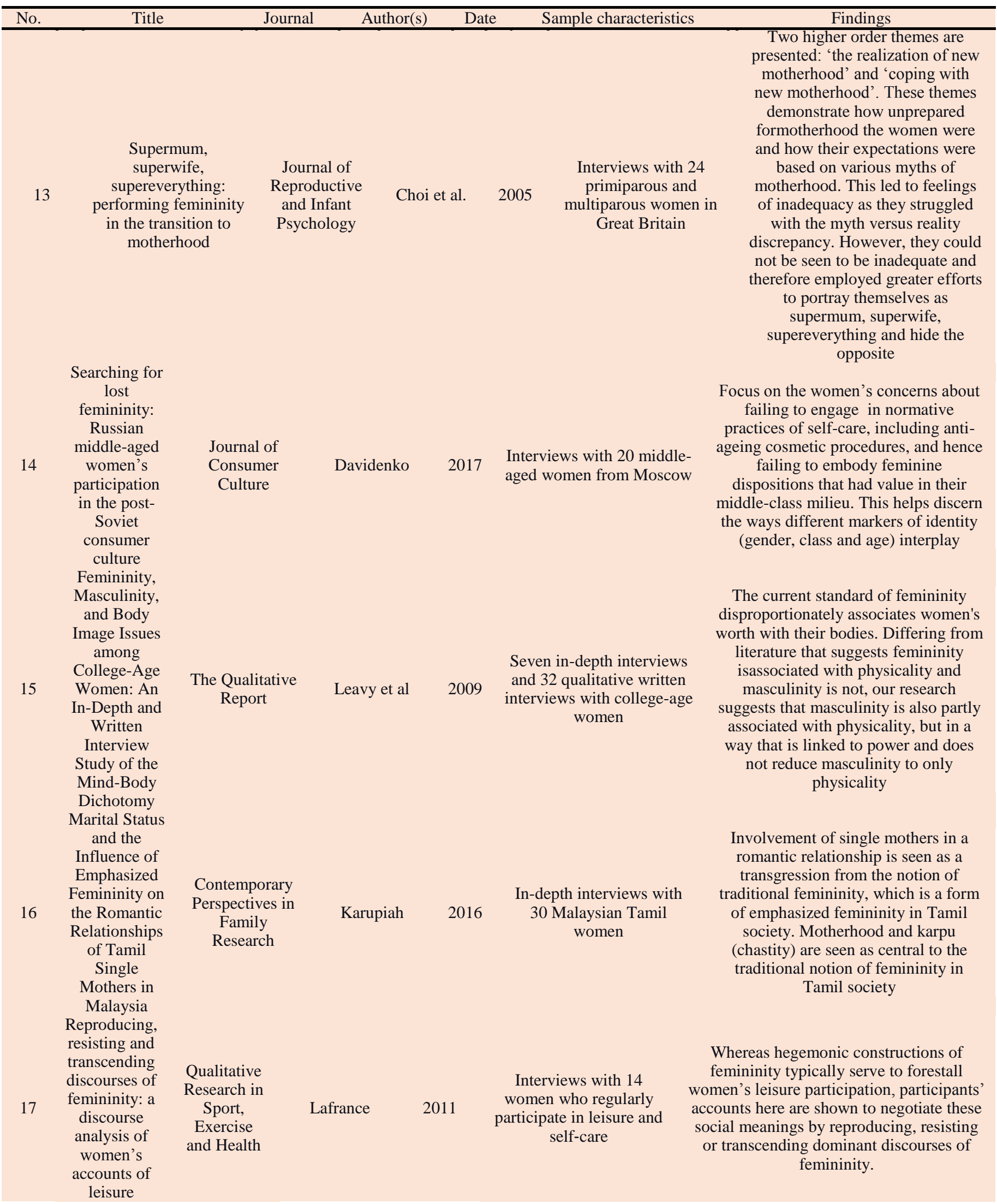




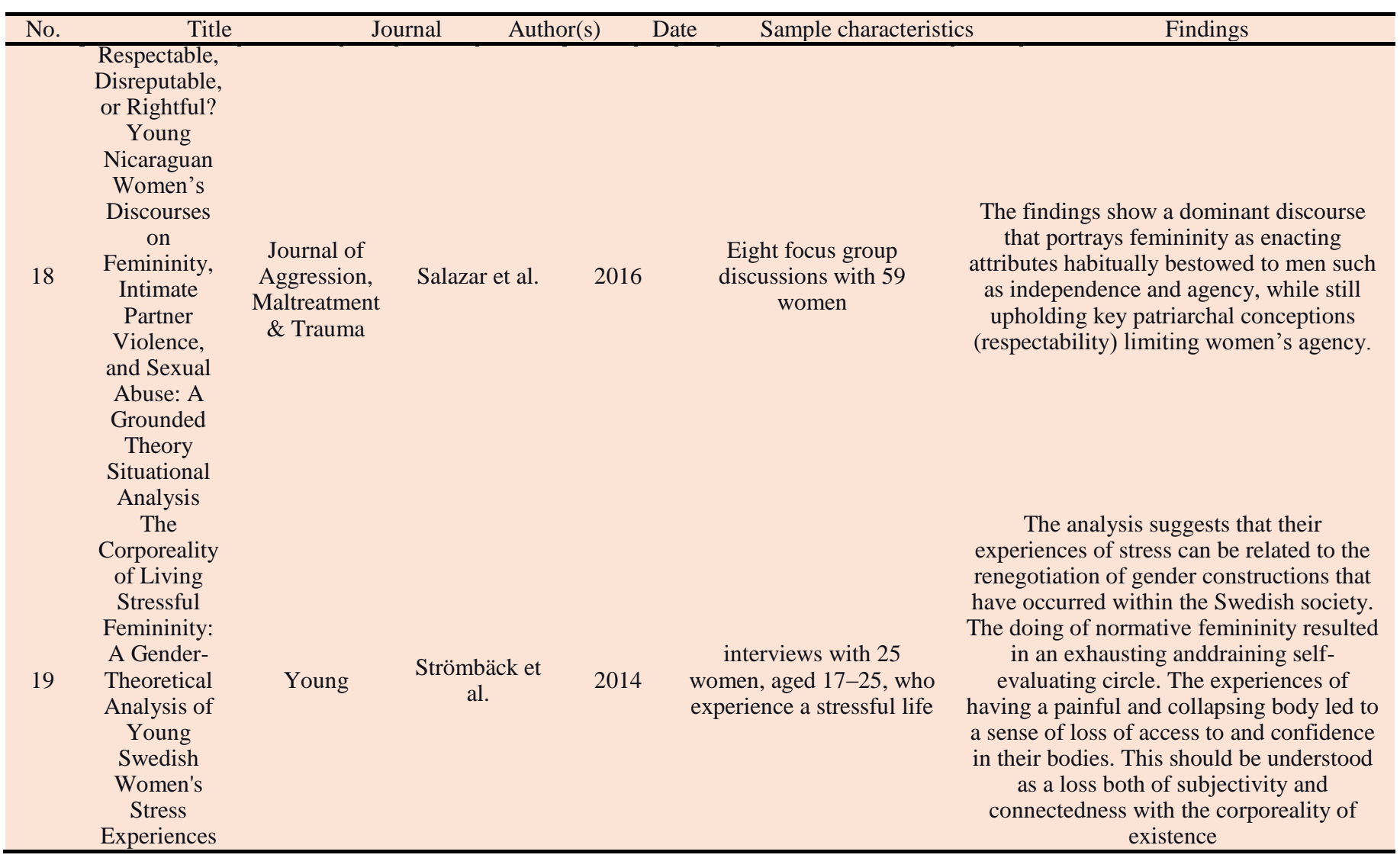

\section{Conclusion}

The present study was conducted using a systematic review method to survey the results of research on the perception of femininity in different countries between 2000 and 2020. From the findings of the researches, six factors were extracted: sexual agency, motherhood, appearance and body, independence and respect, caregiver or self-care, and cultural factor, each of which are discussed here.

Sexual Agency: Two of the studies reviewed in this paper have dealt with the concept of sexual agency in women and femininity in general. Participants in Quach's (2008) study on women in Hanoi, the capital of Vietnam, provided a definition of femininity that emphasized sexual inexperience, obedience, passivity, and low levels of desire. A 2016 study by Kelly et al. in Australia found that women exercised their femininity by focusing on enabling their male partner's pleasure, while ignoring their own sexual desires and aspirations.

The research conducted by Fiaveh et al. (2016) presents different results than previous researches and informs about sexual agency in Ghanaian women and shows that they are discussing sexual risk. In many African contexts (e.g. Ghanaian Ashanti, Senegalese July Muslim, Ugandan Boganda, and Sentoku women in South KwaZulu-Natal), women display extensive sexual negotiation skills and are often sexually expressive.

Motherhood: Choi et al. (2005) argue that marriage and motherhood are central to femininity, and therefore they act like a source for constructing feminine identities as well as providing social and material rewards for women.

In Karupiah (2016), which examines single mothers in Tamil, they identified their responsibilities in such a way that motherhood was the most important part of their lives and involvement in a romantic relationship was a transgression from the notion of traditional femininity.

In both studies, femininity, and especially motherhood, is defined by the characteristic of devotion, self-sacrifice, needlessness, and at the same time, meeting the needs of the spouse and children. Such a woman seem to be passive in meeting her own needs. 


\section{Monthly Journal of Psychological Science}

Appearance and body: In this research review, many studies have addressed the issue of appearance and body and its relationship with femininity. These included the psychoanalytic view of the body, the standard cultural issue of young women, and the middle-aged view of their bodies.

In their paper, "The riddle of femininity: The interplay of primary femininity and the castration complex in analytic listening", Fritsch et al. employed a psychoanalytic view toward body, where penis envy and phallic desire were discussed. They stated that penis envy may be related to an attempt to merge with a pre-Oedipal mother or a desire to please an Oedipal mother.

Leavy's (2009) study of cultural issues faced by college-age women suggests that the current criterion of femininity disproportionately relates women's value to their bodies. Femininity is often associated with physical characteristics, and the "good body" is feminine. The message received from these various sources is unshakable, and young women realize that in order to be considered feminine, they must strive to achieve this cultural standard.

A study by Strömbäck et al. (2014) shows that young women investing in normative femininity, with an emphasis on perfection, progress, and accessibility, creates a grueling and eroding situation that leads to a loss of subjectivity and connectedness with the corporeality of existence.

The Bush's (2000) study of cervical screening, the body and femininity takes a different view of women's bodies and considers it to be a problematic and annoying issue. Women's fears about their bodies are significant as a dangerous place that, if left unchecked, can get out of control.

Rutagumirwa and Bailey (2017) examined the importance of the body in older women, and this study showed that older women perceive their aging body as a "burden". This way of describing the body is due to the inability of the old body to have a life similar to the sexual biographies of women. The conflict between their physical limitations and their desire to perform gendered tasks (internalized feminine habits) affects the process of selfidentification.
Vol. 20, No. 103, Autumn(October) 2021

Davidenko (2017) also focuses her research on middle-aged women and looks at the aesthetics of the middle-aged body. The women in the study saw beautification as a way to take care of themselves and maintain their own unique advantage, but many did not necessarily accept the need for radical reshaping of their bodies. As middle-aged women get older and think about their lives, they may resort to methods other than beauty care (such as psychology) to get the idea that women need to be feminine. They are constantly working on their bodies and questioning their sexual attractiveness from the masculine perspective.

Independence and Respect: Studies that have examined "femininity, respect, and independence" also point to cases in which changes in social, economic, religious, and even immigration conditions have led women to gain greater independence and respect. It also raises the issue that the violent conditions of society have reduced the respect and independence of women. These cases will be discussed in detail.

Mitra's (2013) study, which examines the impact of domestic violence on Indian women, can be seen as a loss of independence and respect for women. Because this violence is experienced in private and imtimate relationships, it causes emotional isolation of women and loss of self-confidence, low self-esteem and high dependence on aggressive husbands.

But other research indicates an increasing trend in women's independence.

Remennick (2007), who studied former Soviet women living in Greater Boston, points out that while a large number of Jewish immigrant women live in the United States (compared to their counterparts in Israel and Europe) can leave employment and become full-time housewives due to the high income of their spouses, few of them choose this option. The need for work experience, self-actualization and economic independence is deeply rooted in the cultural/Russian tradition of the Soviet Union, which includes at least two generations of women.

In their study of Nicaraguan women, Salazar et al. (2016) stated that economic and social change has helped to create and promote an ideal young woman who does not accept passivity and advocates (and to 


\section{Monthly Journal of Psychological Science}

Vol. 20, No. 103, Autumn(October) 2021

some extent seeks) independence, success and resilience of women and girls.

McGinty (2007), in his study of the formation of alternative femininities through Islam, quotes a female Muslim convert in Sweden who believes that converting to Islam creates a new sense of femininity, which is defined by integrity, chastity, and respect, without compromising one's independence and professionalism.

In 2015, Alemdaroğlu published a study titled "Escaping femininity, claiming respectability: Culture, class and young women in Turkey". The narratives of the women interviewed in this study indicate that young women actively distance themselves from other women and escape from certain forms of femininity. While young women escape certain forms of femininity, they use alternative narratives such as independence, career success, moderation, chastity, and religiosity to attest self-value and respectability.

Caregiver or self-care (responsibility for oneself and others): Research in this area has pointed out that the mental assumption of different societies is that the task of caring (oneself and others) is associated with women, which is a psychological pressure for women.

In Charlebois (2010) study, Japanese women expressed their gender identity through the interpretative repertoires of women as natural caregivers, mothers as primary parents, and gender equality. This study witnessed a discourse conflict between "mothers as the main parent" and "gender equality", which shows that their collective wisdom is entering a state of discourse change and reformulation.

In Choi et al.'s (2005) study of women in the UK, in addition to the role of mother and child caregiver, housework and caring others are also assigned to women.

In a study of Canadian women participating in selfcare and leisure programs, LaFrance (2011) referred to a kind of Western patriarchal culture that presents discourses of femininity that make women inherently caring (e.g. Maternal instinct).

Cultural factor: In researches conducted in this field, we can see historical and cultural differences in the definition of femininity. Remennick's (2007) study of Russian immigrant women, mentioned in the section on "independence and respect", refers to the history of the role of women in the Soviet Union in the 1980s, when discriminatory attitudes and behaviors toward women led to lower or middle-class jobs for them. With the economic instability of 1990 and the faltering jobs of men, many women became the only direct breadwinners of the family. Instead, many women, tired of multiple roles, established traditional femininity through a slim and attractive (sexy) look taken from the suburban housewives of American and French films. In this atmosphere in 1990s, many young Russian women who were in their twenties believed that their youth and beauty were the mainstays of investing. The traditional divisions established, and women returned to services, secretarial work, sales, and the beauty industry while leaving business, technology, and finance to men.

Chang et al.'s (2017) study of Asian American women showed that when family conflict is greater, femininity is more likely to be described in terms of obedient roles.

Charlebois (2010) also stated in his work on Japanese women that being at home and family is an essential element of middle class femininity in post-World War II Japan. It is noteworthy that the role of a professional housewife is not seen as a form of imprisonment in house, but is still popular among many women.

Interestingly, the main focus of the mentioned researches is on East Asian women and Russian women, which in fact covers North Asia. However, there are still significant differences in the definition of femininity among these people, and this confirms the importance of understanding the culture and history of a country in examining the concept of femininity.

According to the results of the present study, it can be said that femininity does not include one or two specific factors and there is a set of factors that form the concept of femininity. Identifying and studying these factors can help in the developing programs for women's mental health and research in the field of women's studies. between male and female occupations were re- 
Like any other studies, this study also had its own limitations, the most important of which were the lack of access to the full text of a number of studies, and languages other than English, which inevitably fell out of analysis.

It is suggested that researchers study and compare the concept of femininity in Iranian women who belong to different groups of Kurdish, Turkish, Lori and Persian language, and thus take an important step to study and help women in this region.

\section{Ethical Considerations}

Compliance with ethical guidelines: The authors extracted this article from the $\mathrm{PhD}$ dissertation of the first author in clinical psychology, in the Faculty of education \& psychology, shahid beheshti university, Tehran.

Funding: This study was conducted as a $\mathrm{PhD}$ thesis with no financial support.

Authors' contribution: The first author was the senior author, the second and the third were the supervisors and the forth and fifth were the advisors.

Conflict of interest: the authors declare no conflict of interest for this study.

Acknowledgments: I would like to appreciate my supervisors and advisors. 


\section{ادراكى از زنانكى در كشورهاى مختلف: يكك فراتركيب كيفى}

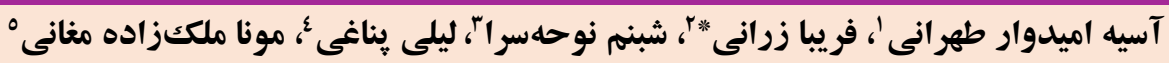

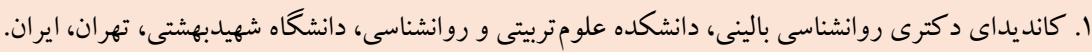

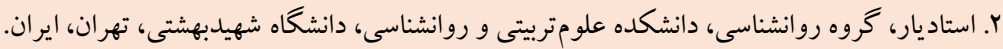

r.

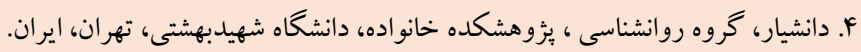

هـ دانشيار، گروه راديوترايى، دانشكده يز شكى، دانشكاه علوم يز شكى شهيدبهشتى، تهران، ايران.

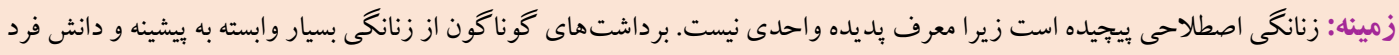

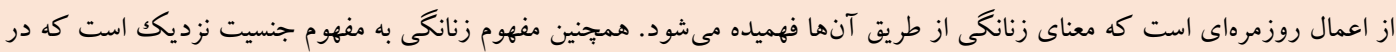

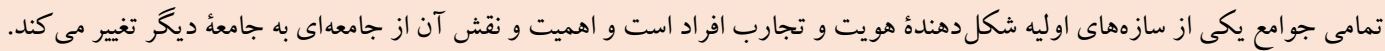
هدف: از آنجايى كه تعريف زنانكى بسته به فرهنگ و استانداردهاى هر كشور متفاوت است، لذا بررسى ادراك زنان كشورهاى مختلف از

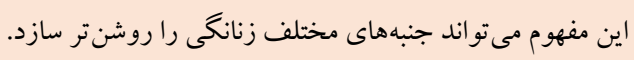
مشخصات مقاله

ادراكك از زنانكى، مادرانكى، فرهنگك،

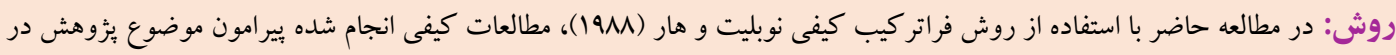

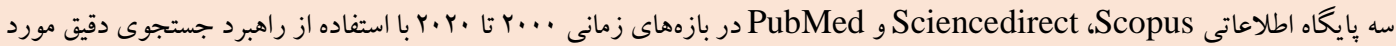
بررسى قرار كر فتند. در نتيجه اين جستجو WVW مقاله بازيابى و بر اساس دستور العمل بريزما و ملاكك هاى ورود و خروج، 19 المقاله براى ورود

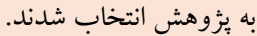
يافته ها: بر اساس ئزوهشهاى مورد بررسى، شش عامل مختلف در زمينه ادراكت از زنانگى بدست آمد: عامليت جنسى، مادرانكى، ظاهر و 
برسد. آنها جنسيت را صرفاً به مثابه تحقق نقش دركى نمى كنند، زيرا برخلاف نقشهاى ديخر (مانند بزشكك، برستار يا بيمار)، جنسيت به تمام نقشها و موقعيت هاى اجتماعى رسوخ مى كند (كلى و همكاران، 19 بـ (Y).

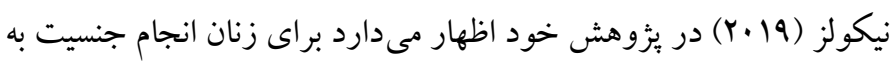
معنى انجام موفقيت آميز زنانكى است، به اين معنى كه زنان صفات، حالات

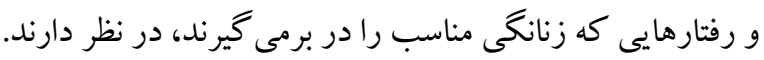

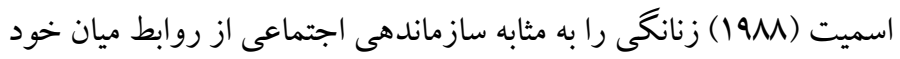
زنان و ميان زنان و مردان تعريف مى كند كه توسط متنهاء؛ يعنى اشكال به رانه

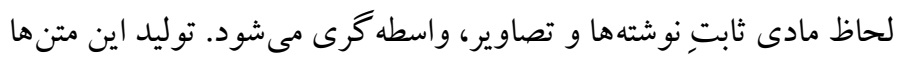

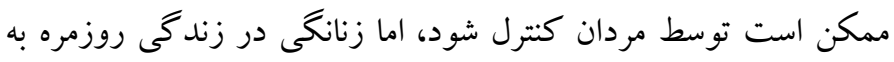

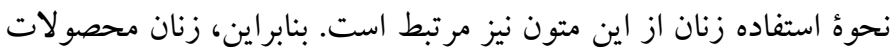

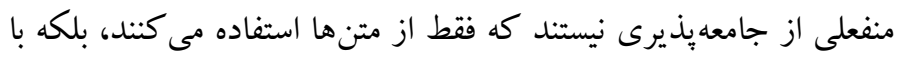

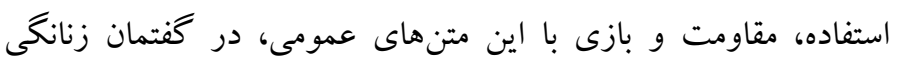

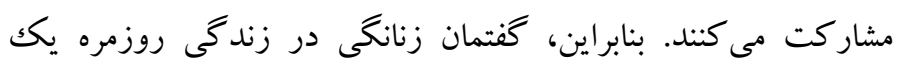

سازماندهى اجتماعى جارى و در حال تكامل است (كاروييا، (Y) 19).

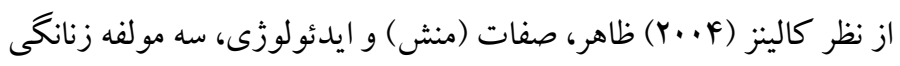

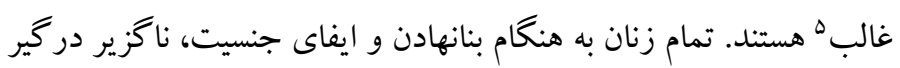

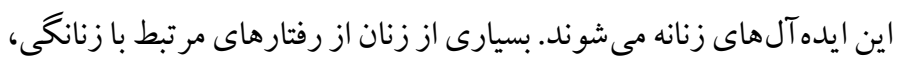
على الخصوص ظاهر و مادرانكى، لذت يا قدرت كسب مى كنيند. ايجاد و

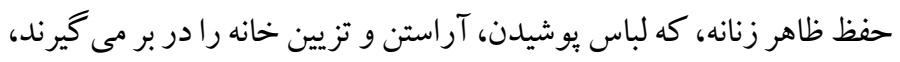

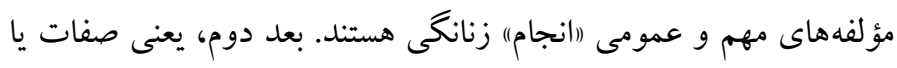

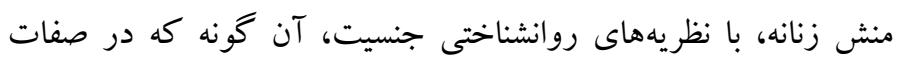

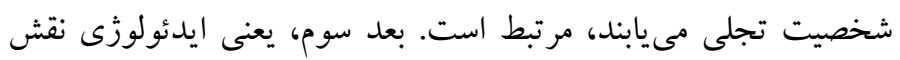

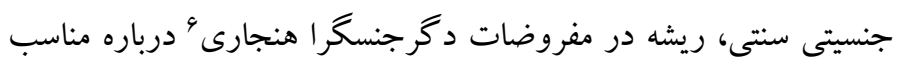
بودن و مطلوب بودن نقشهاى مجزا و تعيين شده براى زن و مرد دارد، كه رهن

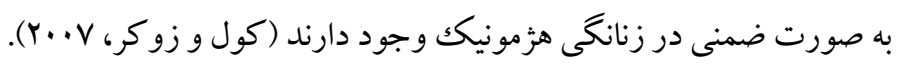

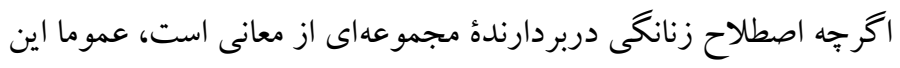

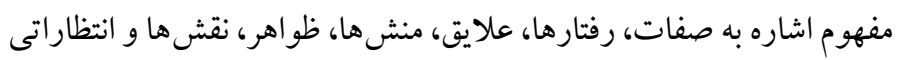

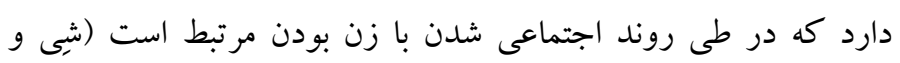

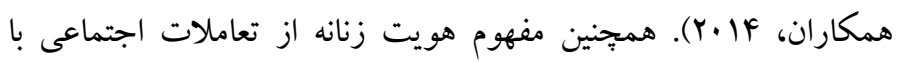

${ }^{4}$. texts

${ }^{5}$. dominant femininity

${ }^{6}$. heteronormative
مقدمه

دو كانهُ مردو وزن از ابتدايى ترين دوتايىهاى بشر است كه بر اساس تفاوت زيستى بنا شده است و مربوط به حوزه جنسيت مى شود. آنجه از دو مفهوم

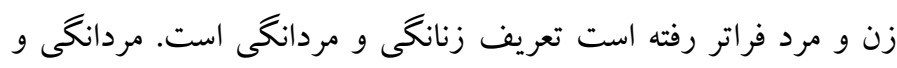

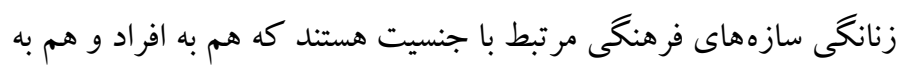

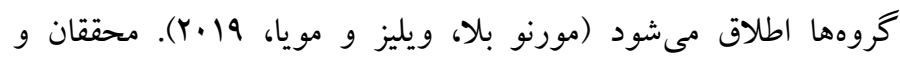

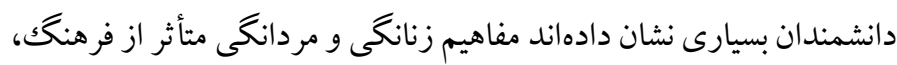

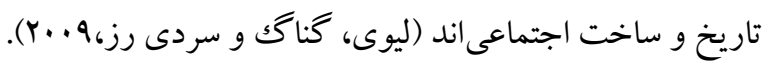

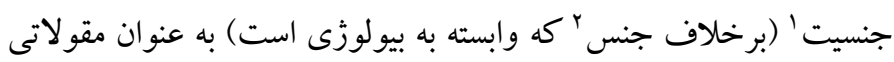

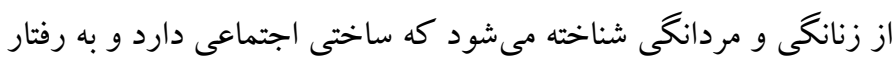

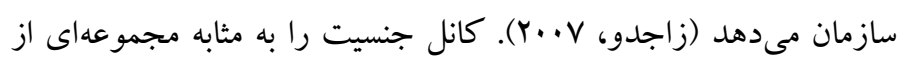
روابط سلسلهمراتبى، ساخته شده به لحاظ اجتماعى و سيال مفهوميردازى

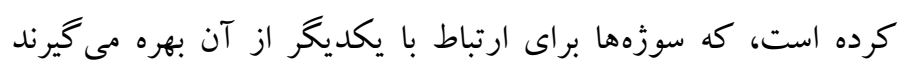

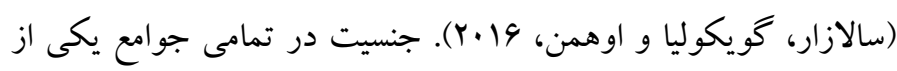

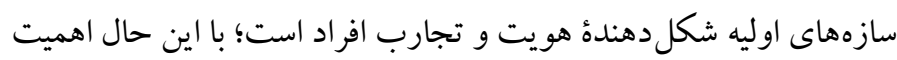
و نقش آن از جامعهاى به جامعهى ديخر و از فرهنكى به فرهنكى ديخر تغيير مى كند. جنسيت يكى از اولين طبقهبندىهاى اجتماعى است كه امروزه

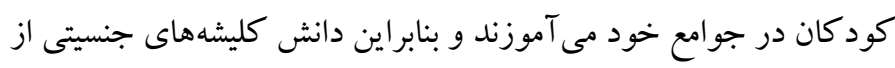

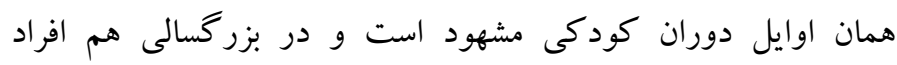

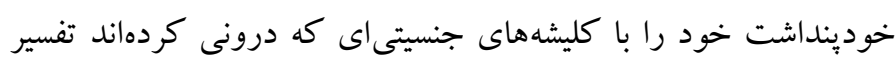
مى كنند (كاجل، استفنز و نيدليج، 19 (r). اين انتظارات جنسيتى از طريق

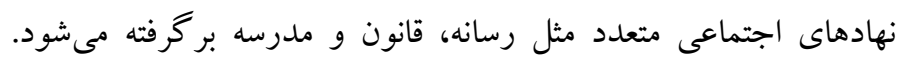

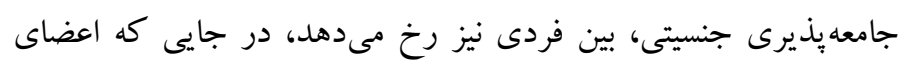

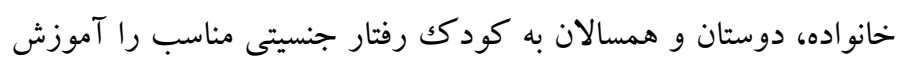

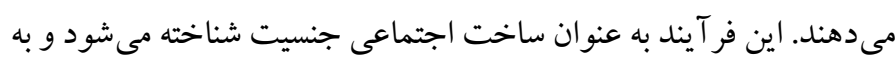

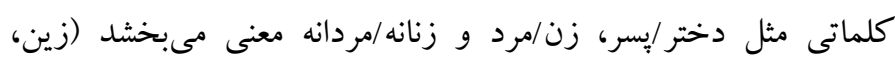

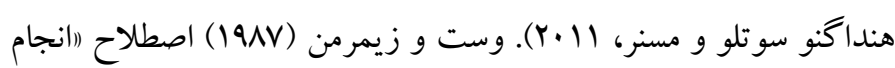

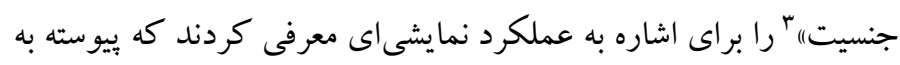
صورت خود آكاه تعديل مى شود و موجب مى شود تا جنسيت طبيعى به نظر

1 . gender

2. sex

3 . doing gender 
زن، اشتراكاتى مثل جهت گیرى به سمت موفقيت، داشتن صفات زنانه،

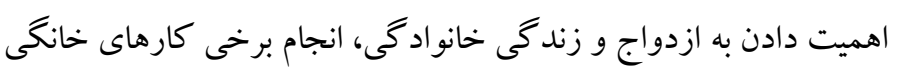
و سرمايه گذارى در ظاهر بين ادراكات زنان گروههاى مختلف يافتند. وجود هنجارهايى همجون نجابت، حفظ آبرو، ديندارى و احترام به دران

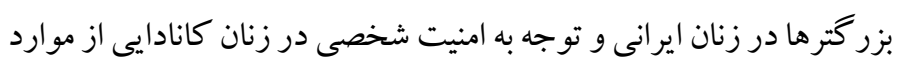

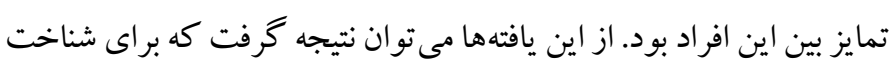

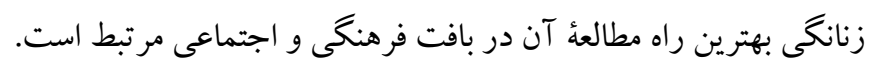

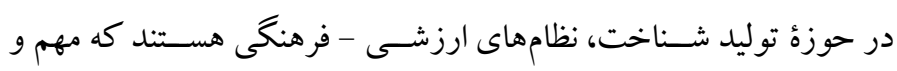

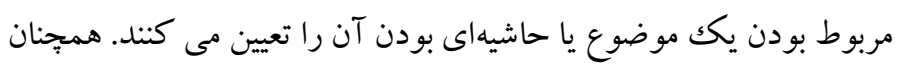

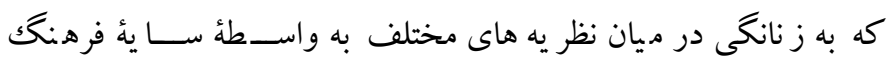

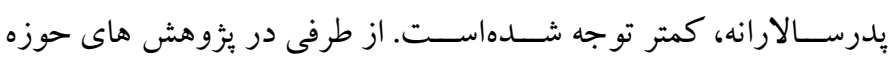
مطالعات زنان نيز به عنو ان بخشـى از مطالعات علوم انســانى، به مطالعه در

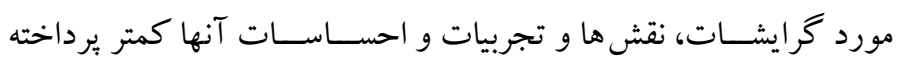
شدهاست. اين در حالى است كه تا وقتى شناخت دقيق و درستى از آنجه آنه كه در ذهن و روان يكك زن نسـبت به بخش هاى ز نا نهى خود موجود

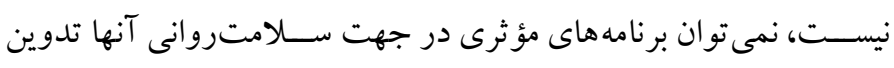

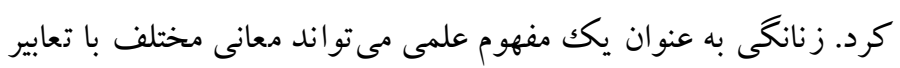

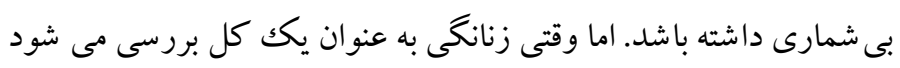

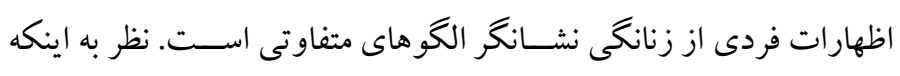

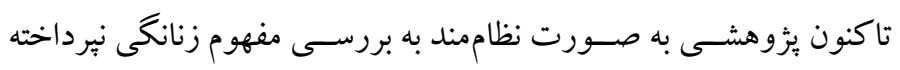

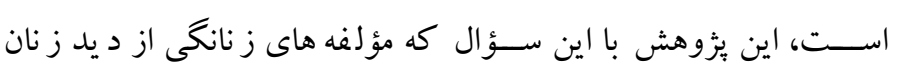
كشـورهاى مختلف جيسـت بـ در صــد اسـت مرورى بر معناى متفاوت

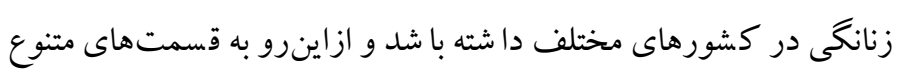
اين مفهوم وسيع از نغاه زنان بيردازد.

روش در مطالعه فراتركيب حاضر كه به صورت سيستماتيك صورت

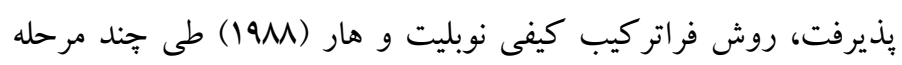

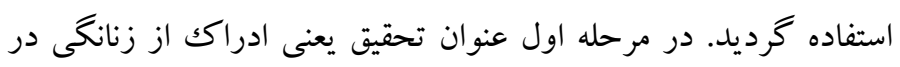

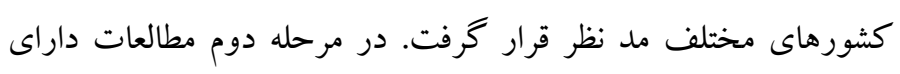

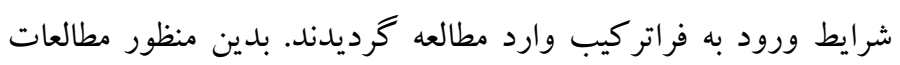

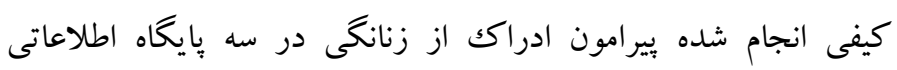

ديخر ان نيز برداشت مىشود. زن خود را با هنجارهاى اجتماعى از جمله

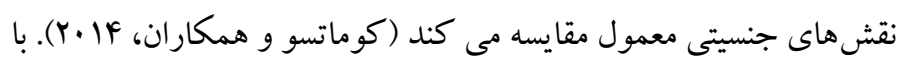

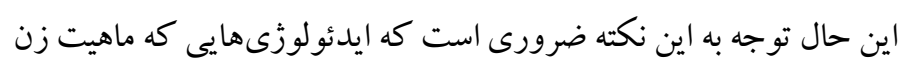

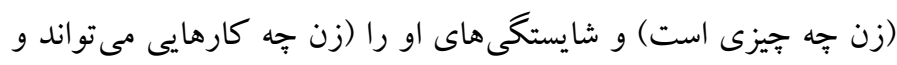

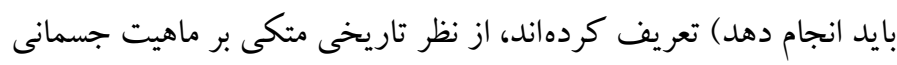

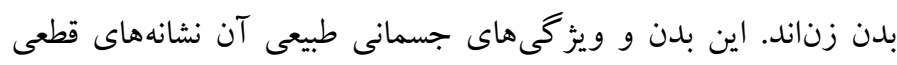

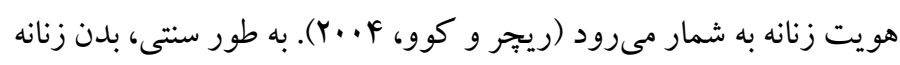

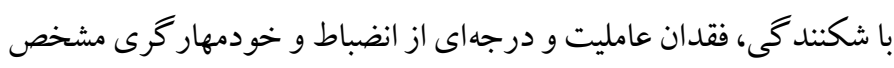

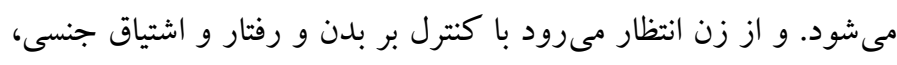

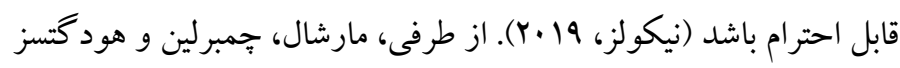

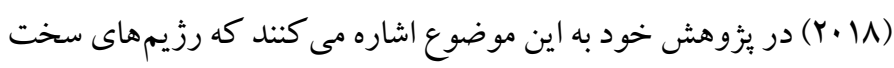
و تمرينات ورزشى تبديل به فعاليت هاى اخلاقى براى زنان شده است كه به إنه به عنوان يك راه اساسى براى كسب بدن ايدهآل از زنانكى نرمال تجويز شده است. زنانكى مانند زيبايى اغلب در جشم بيننده است (كويلن فابى و ويلت،

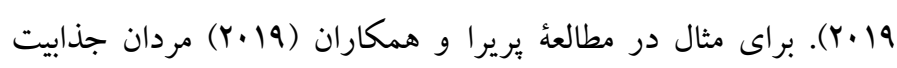

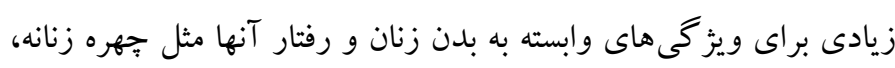

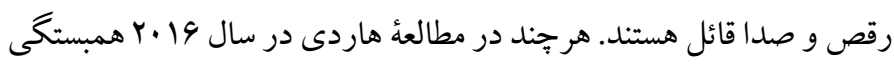

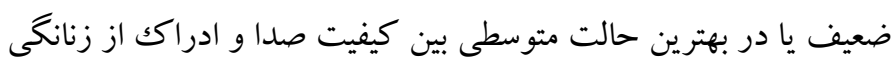

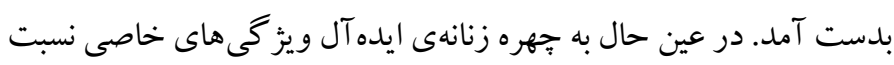

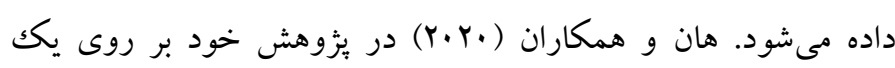

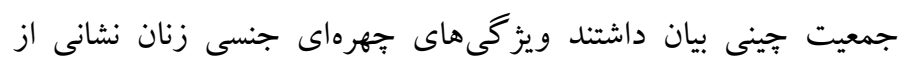

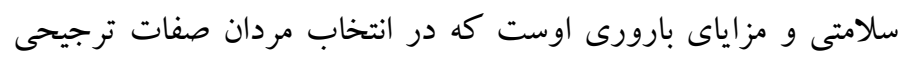

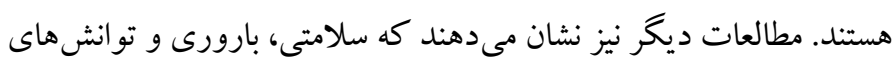

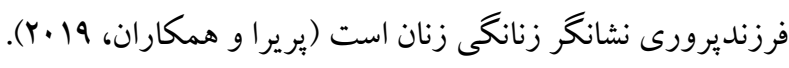

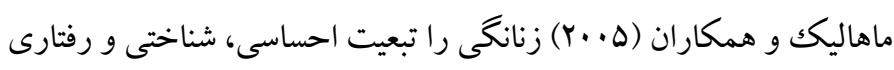

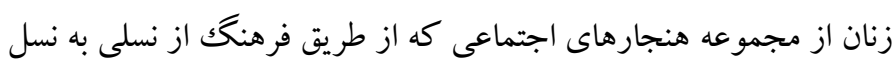

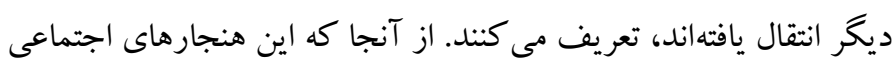

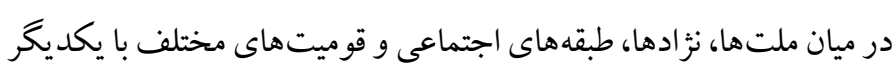

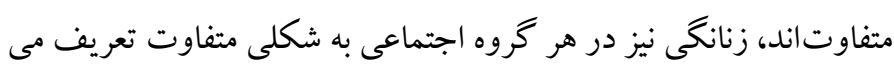

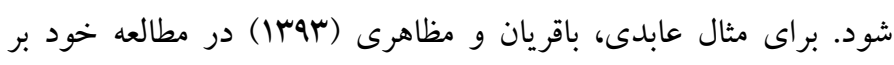

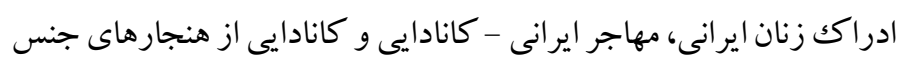


مقايسه شد و در نهايت در مرحله ششم، نتيجه نهايى فراتر كيب يا به عبارت

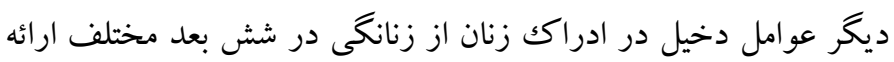
كرديد.

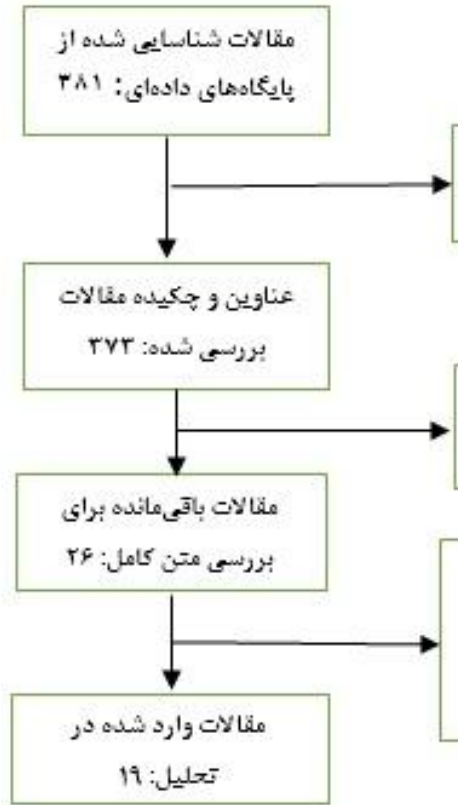

متف به دليل تكرارى بودن

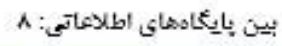

شكل ا. فر آيند بروسى و انتخاب مقالات (درخت تصميم كيرى)

يافته ها اطلاعات مقالات منتخب و يافتههاى آنها در جدول ذيل آمده است. بررسى عواملى كه در اين نوزده مقاله آمده است در شش حيطه اصلى عامليت

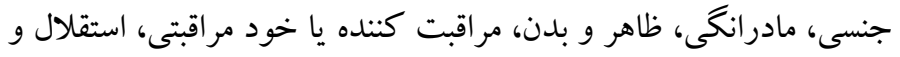

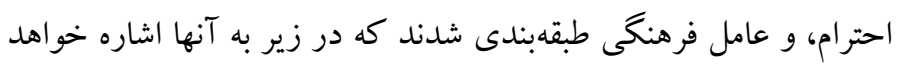

PubMed و Sciencedirect ، Scopus با استفاده از تر كيبى از كليد وازه هاى زير جستجو شدند: Femininity, feminine identity, being woman, perceived femininity

در اين مرحله معيارهاى ورود به مطالعه عبارت بودند از: مقالاتى كه به

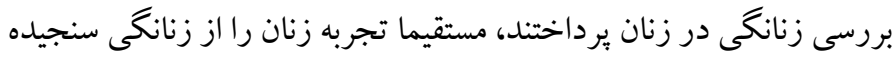

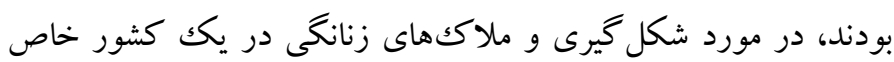

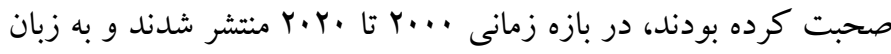

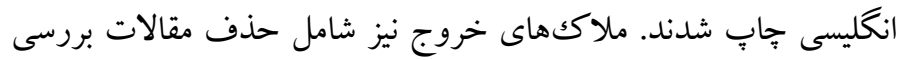
زنانكى در بيمارىهاى جسمى مختلف يا اختلالات روانشناختى خاص،

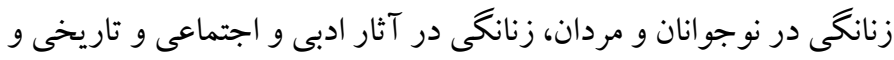
نيز حذف مقالاتى كه متن كامل آنها در دسترس نبود.

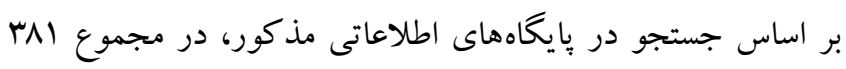

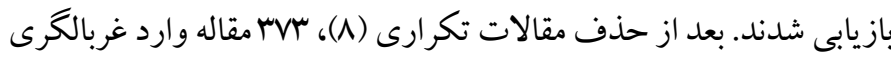

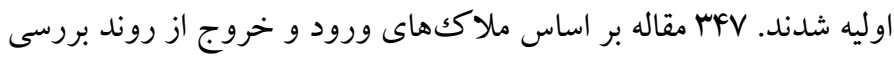

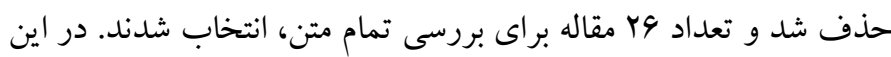

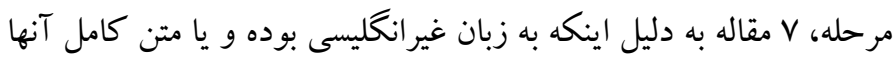
در دسترس نبود، از روند يثوهش حذف و در نهايت 19 مقاله وارد تحيل نهايى شدند (شكل (1). در مرحله سوم، هريكك از مطالعات انتخاب شده، با دقت و تمركز بر

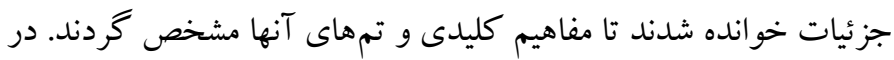

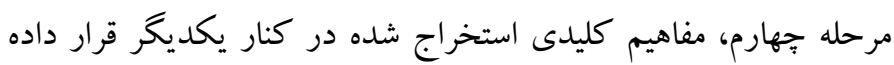

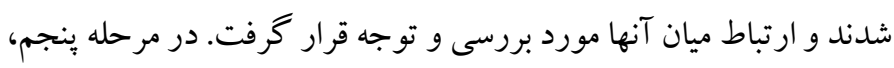
مفاهيم كليدى استخراج شده از هر مطالعه با مفاهيم كليدى ساير مطالعات

جدول 1.اطلاعات مقالات وارد شده در فراتر كيب

\begin{tabular}{|c|c|c|c|c|c|c|}
\hline يافتهها & ويزگ گى هاى نمونه & سال انتشار & نويسند & نام مجله & عنوان مقاله & رديف \\
\hline 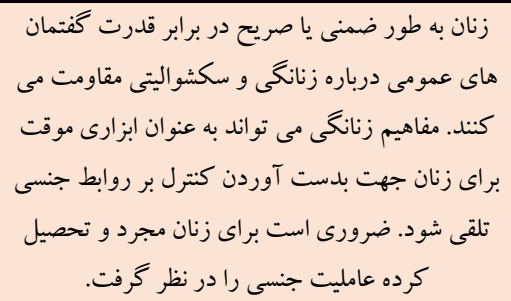 & 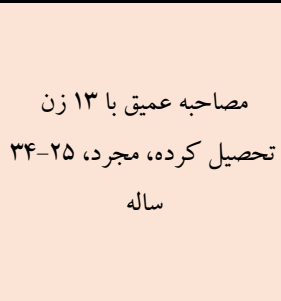 & $r \cdots 1$ & كواج & $\begin{array}{l}\text { Culture, Health } \\
\text { \& Sexuality }\end{array}$ & $\begin{array}{l}\text { زنانكى و عامليت جنسى زنان جوان مجرد } \\
\text { هانوى }\end{array}$ & 1 \\
\hline 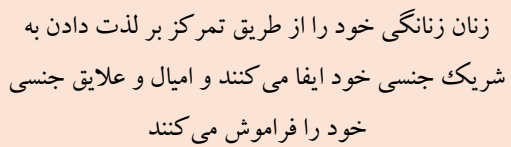 & 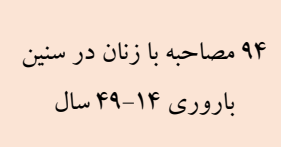 & r.19 & همكار ان و & $\begin{array}{l}\text { Culture, Health } \\
\text { \& Sexuality }\end{array}$ & 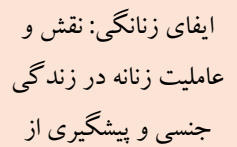 & r \\
\hline
\end{tabular}




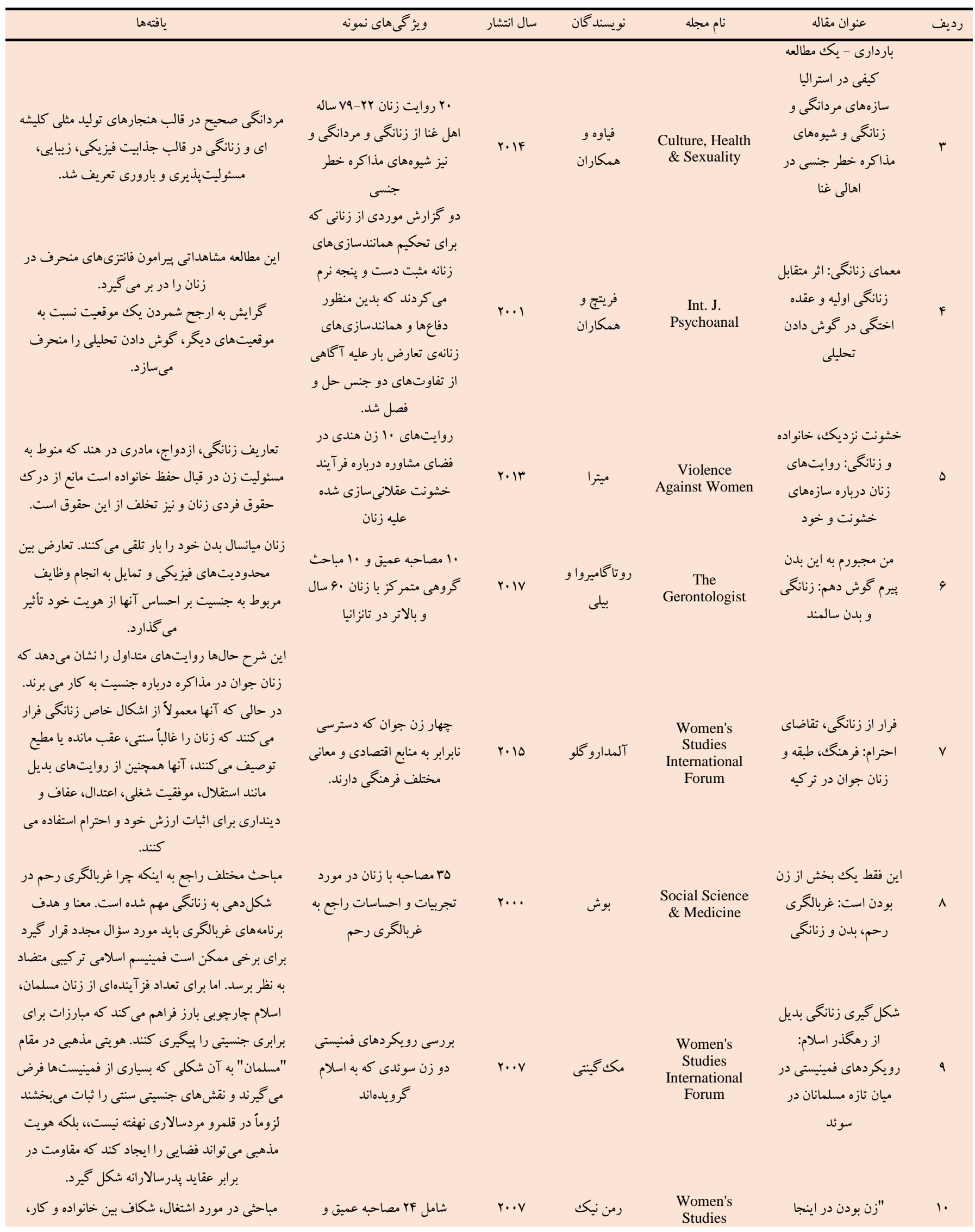




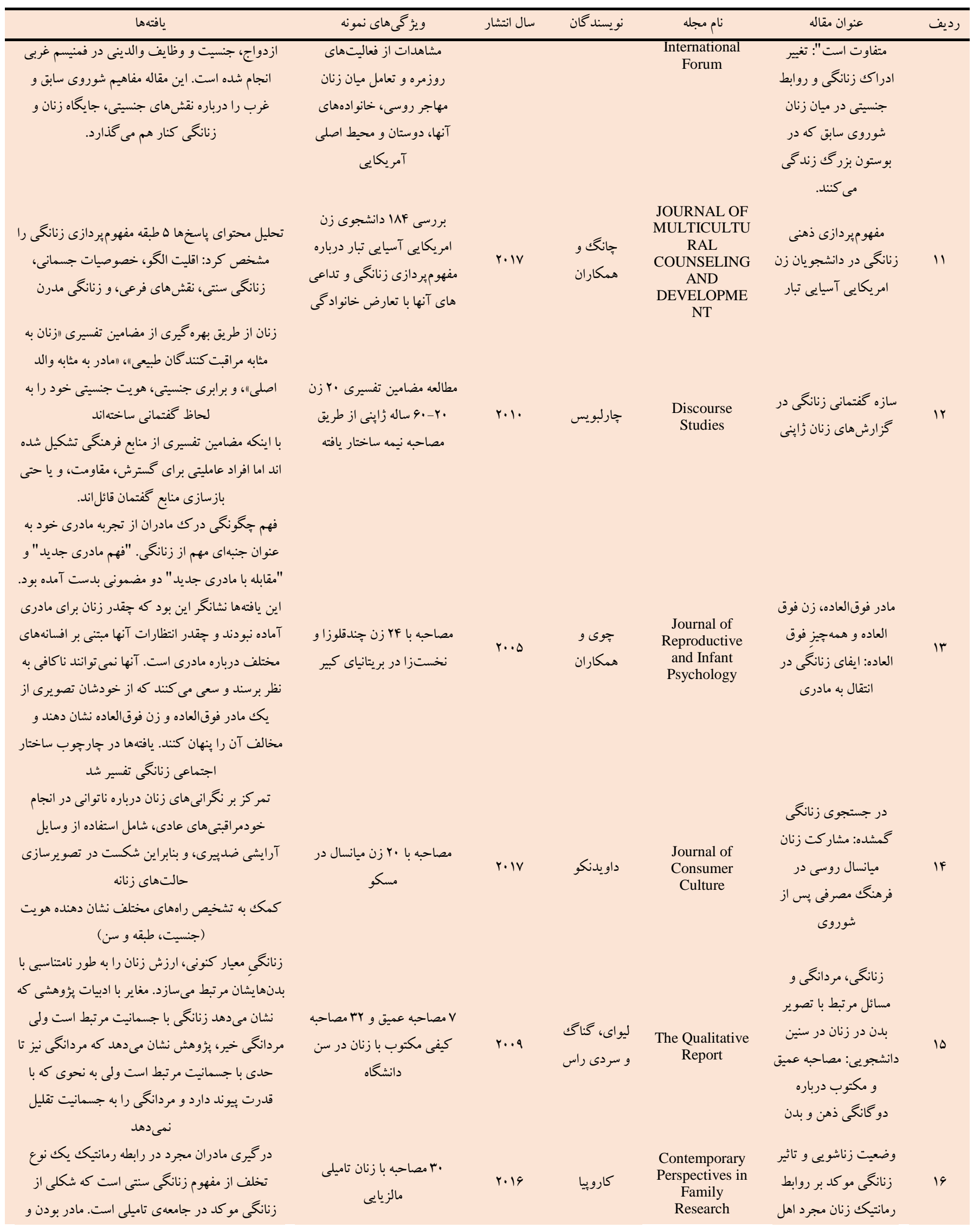




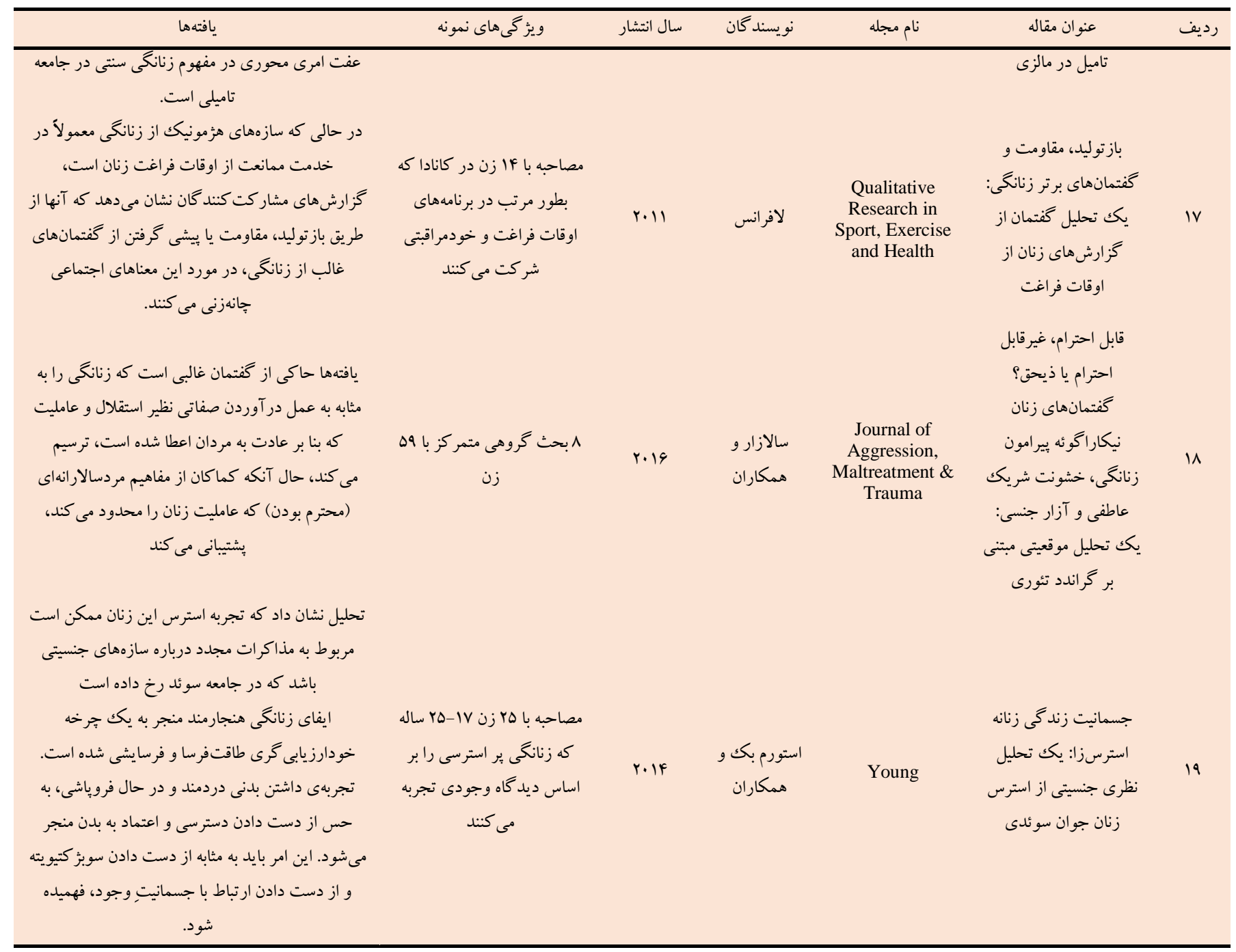

كردند كه بر بى تجربحى جنسى، اطاعت، انفعال و ميل اندكك تأكيد داشت. به نظر مىرسد كه ديد كاههاى زنان دربارهٔ سكسواليته زنانه توسط عقايد فرهنگى مردسالارانه محدود مىشود. زنان جوان براى تصميم گيرى در

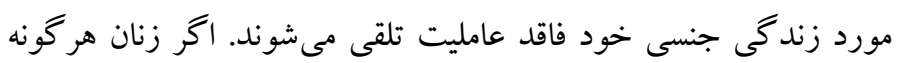
ارزشى براى سكسو اليته شان قائل شوند، اين امر كالايى است كه به براى مردئ مردان واجد ارزش است. مطالعه كلى و همكاران در سال 19.r در استراليا نشان مىدهد زنان با بان تمركز بر ممكن ساختن لذت شريكك عاطفى خود، زنانكى شان را را ايفا دهان

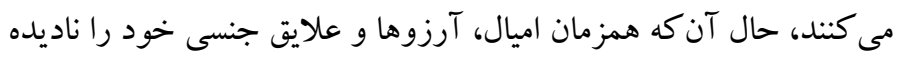

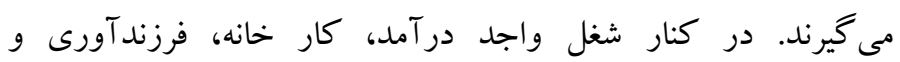

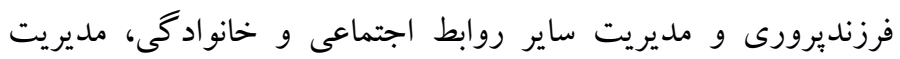

\section{بحث و نتيجه تيرى}

مطالعه حاضر با روش مرور نظاممند به منظور بررسى نتايج تحقيقات انجام -

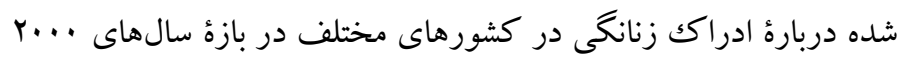

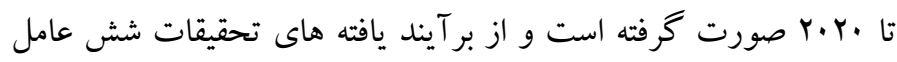

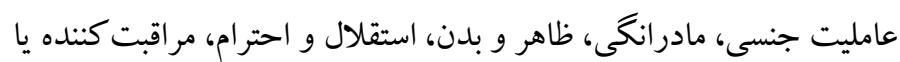

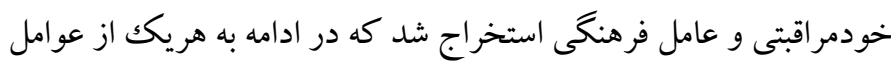
به تفضيل برداخته مى شود: عامليت جنسى: دو مورد از يزوهش هايى كه در اين مطالعه مورد بررسى

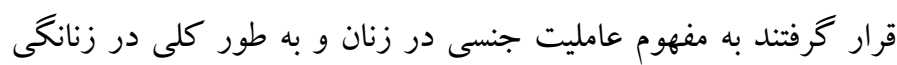

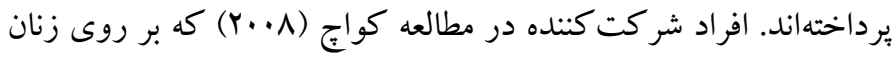
اهل هانوى (يايتخت ويتنام) صورت گر فته است، تعريفى از زنانكى را ار ارائه 
مىشود كه ابرمادر و ابرهمسر را به تصوير مى كشد و متضاد آنها را بنهان

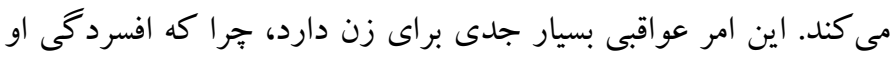
ينهان است. در يثزوهش كاروييا (Y.19) كه به بررسى مادران تكك سريرست در تاميل مى يردازد، مادران مجرد مسئوليتهاى خود را به نحوى مشخص كردند كه مادر بودن به عنوان مهمترين بخش زبند ماردى ماندان بود. بسيارى از آنها

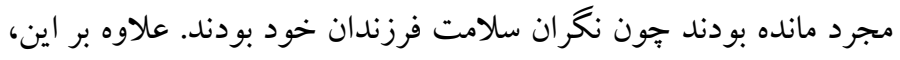

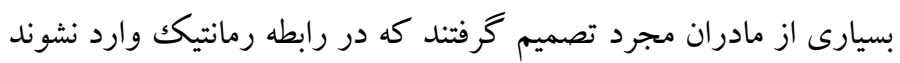

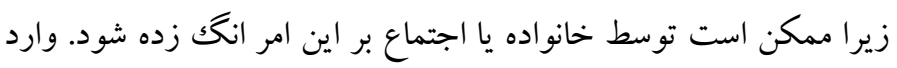
شدن در رابطه رمانتيك به مثابه تخطى از مفهوم زنانكى سنتى تلقى مى شود.

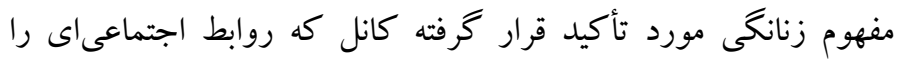
تو صيف مى كند كه شامل اطاعت از مردان است و علايق و اميال آنها را در درد خود جاى مىدهد و نيز بر اطاعت، برورش، همدلى و فداكارى به عنوان

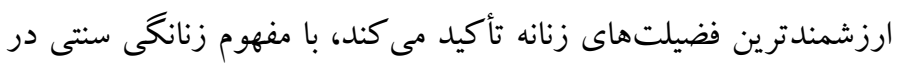

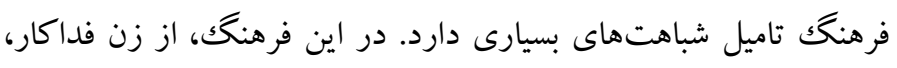
دلسوز و مطيع، اغلب به عنوان همسر و مادرى "ايدهآل)" تقدير مىشود. فداكارى او معطوف به همسر و فرزندانش است. اين خصوص وصيات

بارزش ترين و محبوب ترين ويز گیىهاى زنانه در جامعه تاميل هستند.

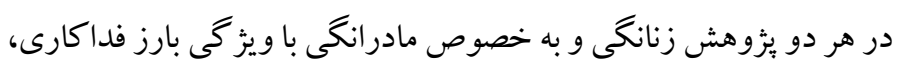

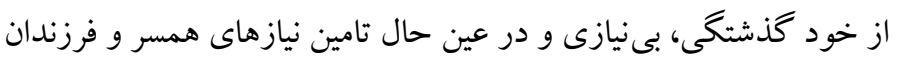

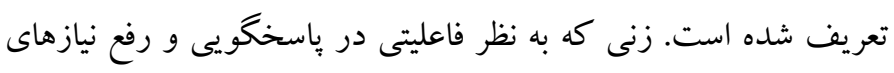
خود ندارد. ظاهر و بدن: در اين مرور بثرهشى مطالعات زيادى به مسأله ظاهر و بدن و ارتباط آن با زنانكى برداخته بودند. نگاه رو انكاوانه به مسأله بدن، مسأله

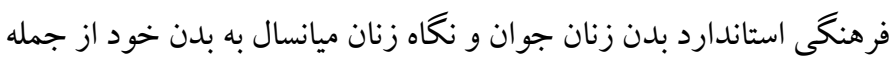
اين موارد بودند. فريتج و همكارانش در مقاله خود با عنوان معماى زنانكى: اثر متقابل زنانكى اوليه و عقده اختخى در گُش دادن تحليلى، نگاهى روانكاوانه به مسأله بدن داشتند كه در آن به رشكك آلت مردانه و داشتن فالوس اشاراتى كردند. رشك آلت مردانه ممكن است به كوششى براى هم آميختكى ' با مادر

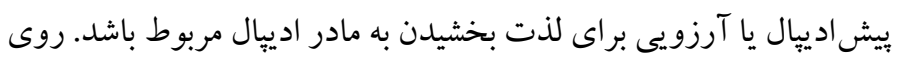

2. merge

$1 \cdot r y$
روش هاى مدرن بيشگيرى از باردارى و زندگى جنسى خوب نيز از جمله

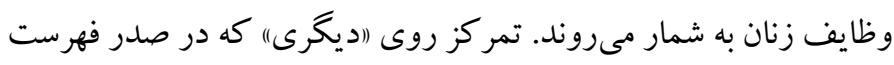

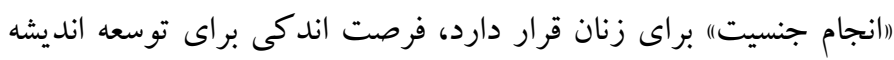

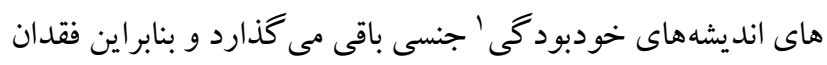
عامليت جنسى در زنان مشاهده مى شود.

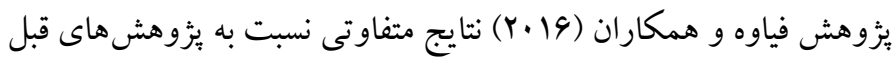
ارائه مى دهد و خبر از عامليت جنسى در زنان اهالى غنا دارد و نشان مى دهد

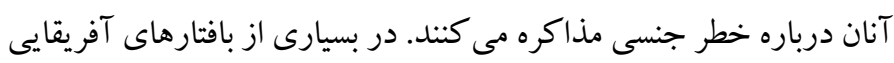

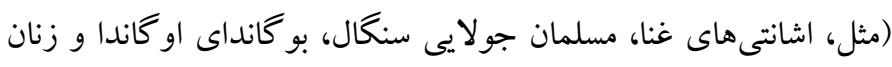
سنتوكوى در استان كوازولو-ناتال جنوبى)، زنان مهارتهاى مذاكره

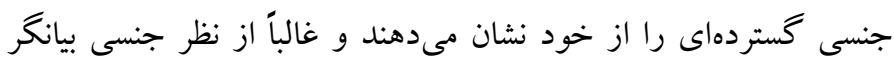
هستند. اكرخه روابط قدرت ساخته شده به صورت اجتماعى، بر شرايطى كه زنان در روابط جنسى شر كت مى كنند تأثير مى گذارند، تحقيقات نشان مىدهد كه زنان در انتخابهاى جنسى خودانديشيده وارد مى شوند.

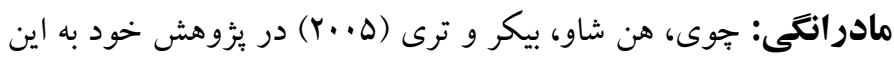

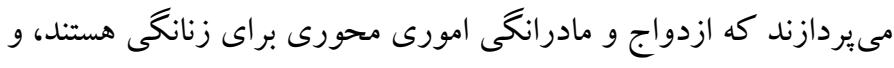

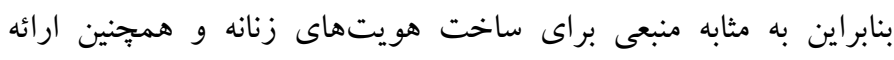
ياداش هاى اجتماعى و مادى براى زنان هستند. لذا عامليت زنان در انتخاب

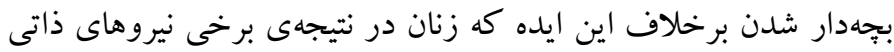

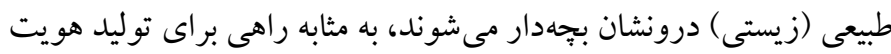

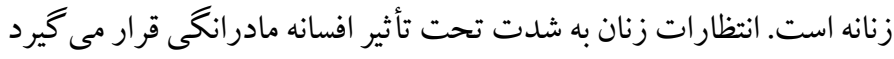

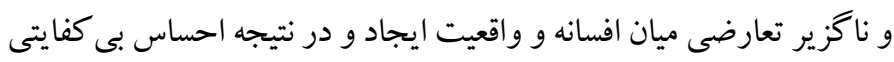

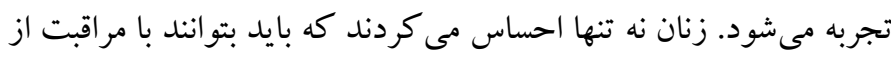

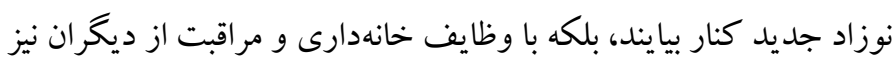

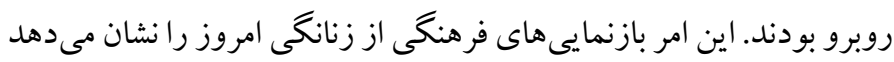
كه زنان را (ابرهمسر) و قادر به كنار آمدن با بسيارى از مطالبات متعارض

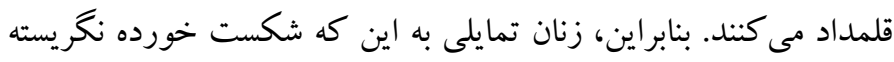

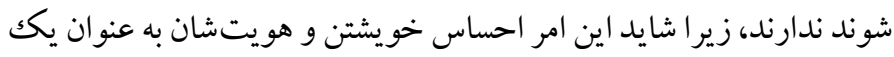

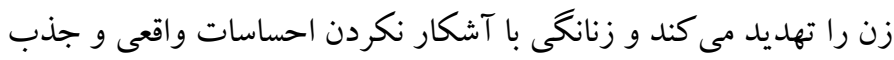

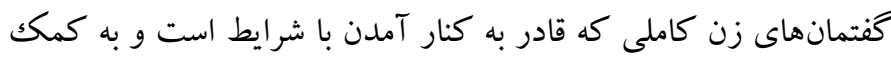

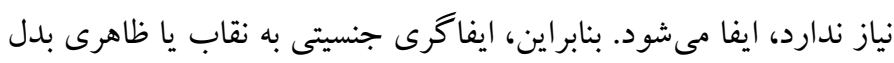

\footnotetext{
1. selfhood
} 
زنان مورد مطالعه اين بثزوهش، انجام تست و آزمايشات زنانه را بخشى از

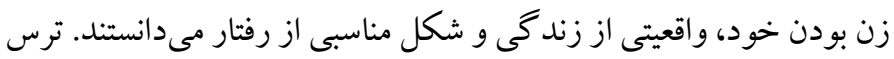

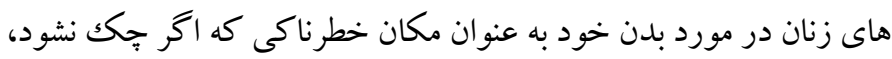
ممكن است از كنترل خارج شود، قابل توجه است. بسيارى از زنان دليل انجام تست را آرامش ذهنى خود ياد مى كنند.

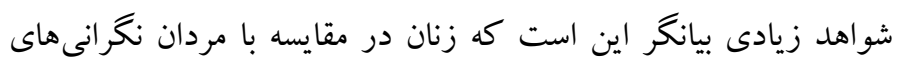
بيشتردر مورد تصوير بدن بدون در نظر گرفتن سن تجربه مئى كنند

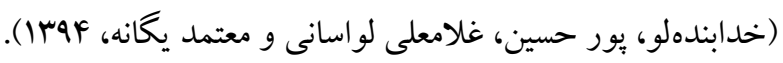

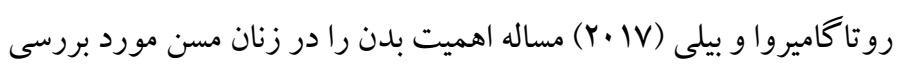

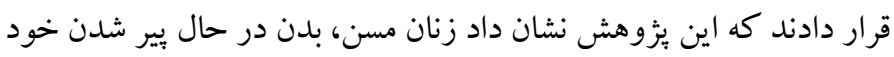

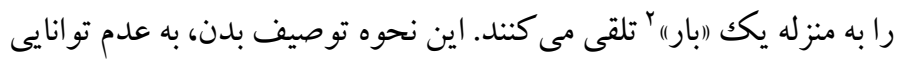

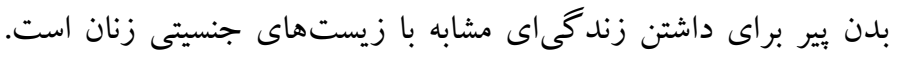

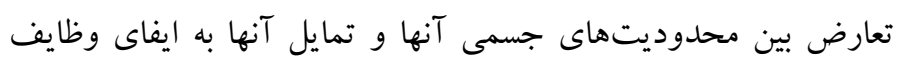

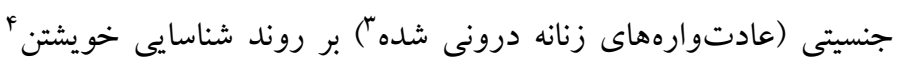

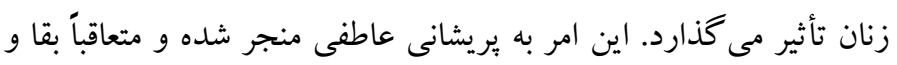

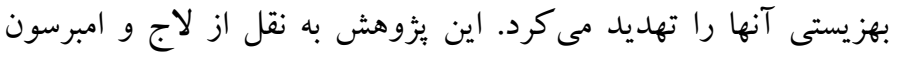

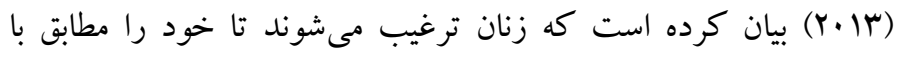
استانداردهاى جوانى ارزيابى كنند، و با افزايش سن احساس شرم و كناه

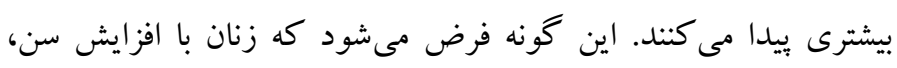

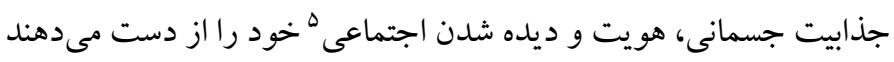

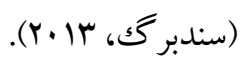

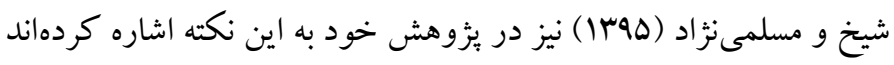
كه اكرجه اين طور فرض مى شود با افزايش سن اهميت جذابيت ظاهرى

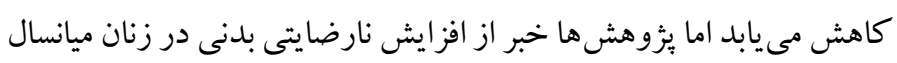

داويدنكو (Y.IV) نيز يزوهش خود را متمر كز بر زنان ميانسال و با نگاهى

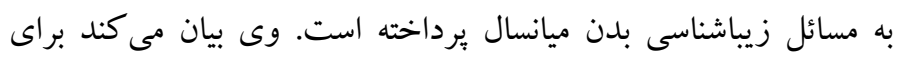
دورانى طولانى مشغوليت زنان به بهبود ظاهرشان، تنها وسيله آنها براى ابراز

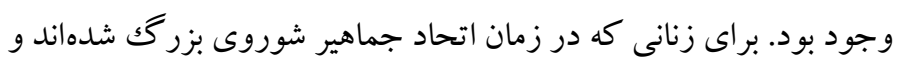

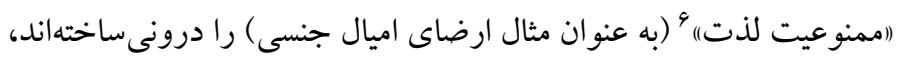

${ }^{4}$. self-identification

5. social visibility

${ }^{6}$. prohibition on pleasure
آوردن به آرزويى براى داشتن يكك فالوس ممكن است دربردارنده گذر كردن دفاعى از اضطراب اندام تناسلى زنانه باشد كه دربردارنده تعارضات

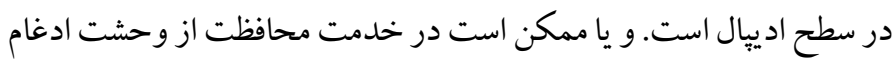

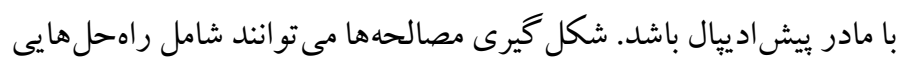

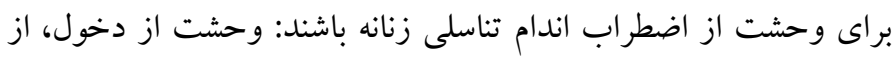
دست دادن لذت يا از دست دادن بارورى.

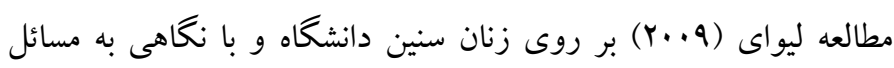

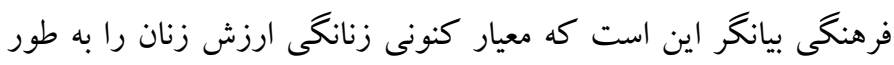

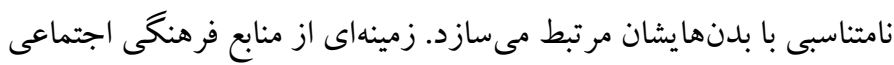

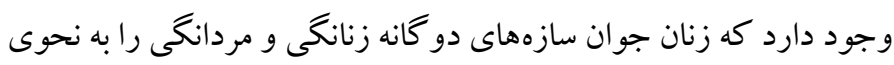

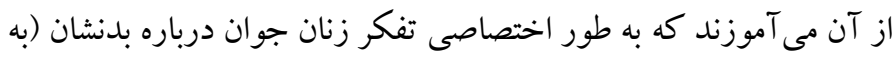

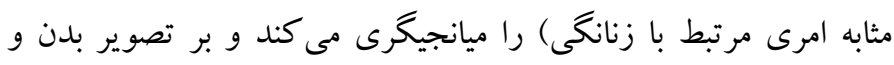

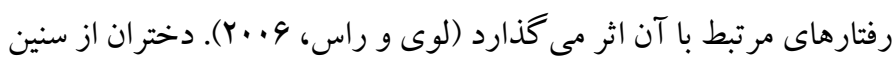

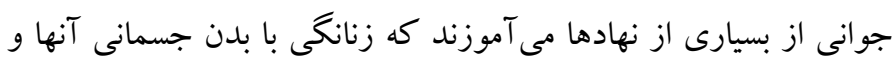

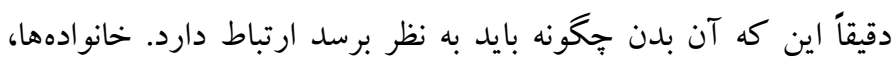

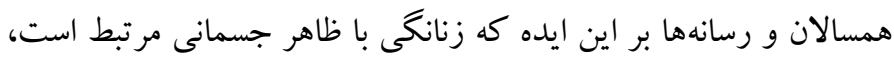

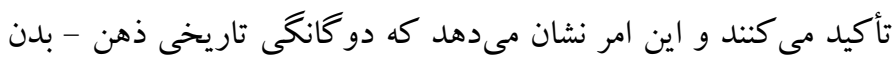

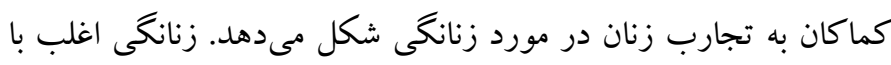
خصوصيات بدنى مرتبط است و (بدن خوب) زنانه است. ييام دريافت شده

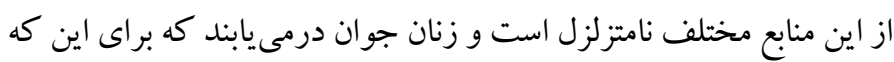

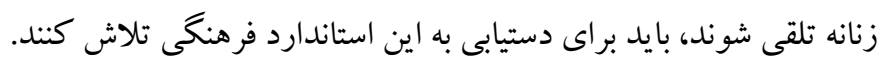

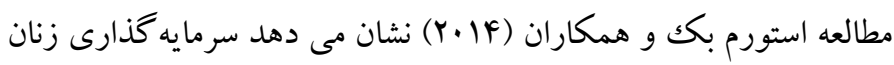

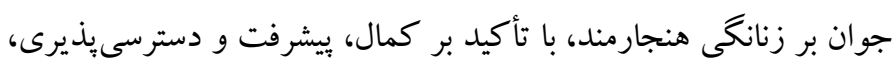

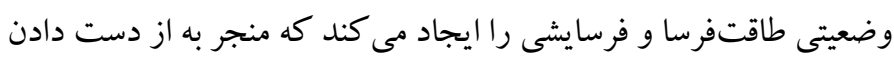

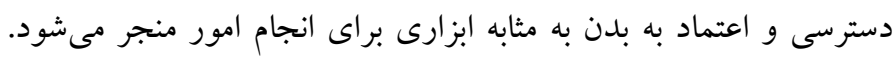

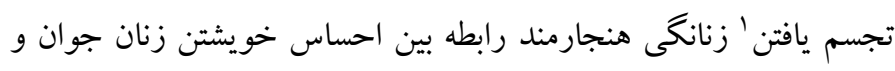
بدنشان را تحت تأثير قرار مىدهد.

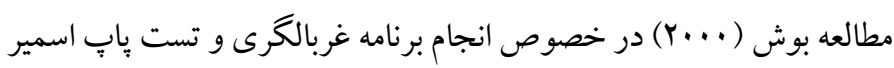

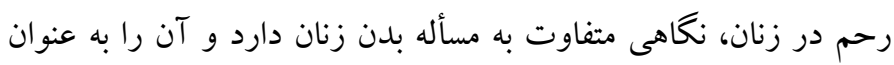

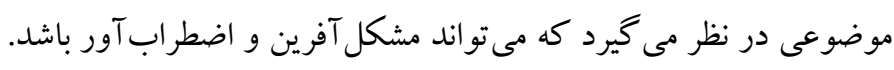

1. embodiment

2 . burden

${ }^{3}$. internalized feminine habitus 
مشترك است، زنان با محدوديتهاى ديخرى روبرو مىشوند. تفاوتهاى

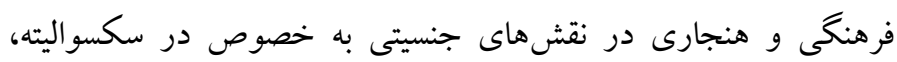

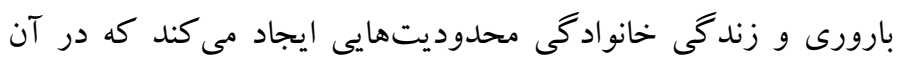

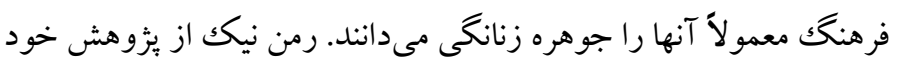

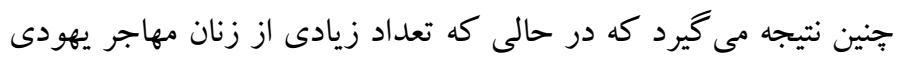
ساكن ايالات متحده آمريكا (در مقايسه با همتايان خود در اسر ائيل و ارويا)

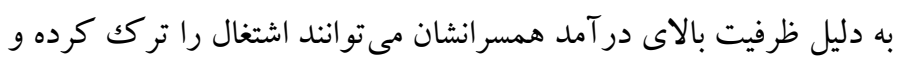

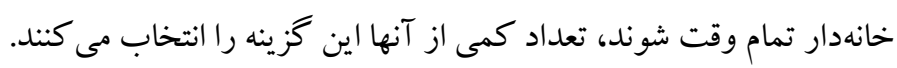
نياز به تجربه شغلى، خودشكوفايى (خوديرورانى) و استقلال اقتصادى عميقاً

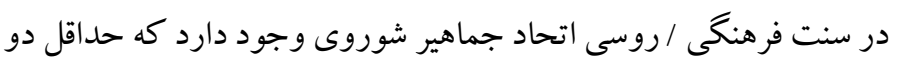
نسل از زنان را شامل مىشود.

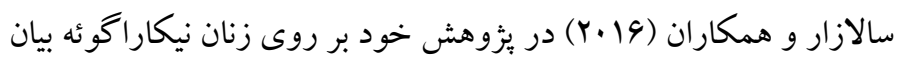

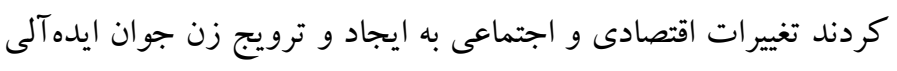

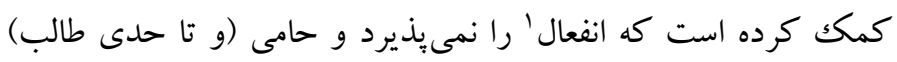

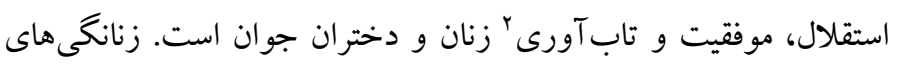

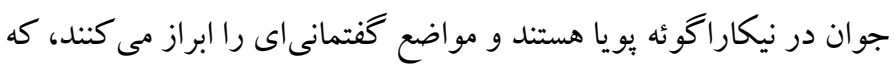
در دفاع از استقلال زنان، برابرى جنسيتى، برهيز از خشونت و احترام با هم تفاوت دارند.

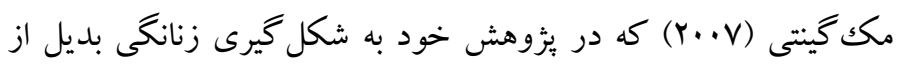

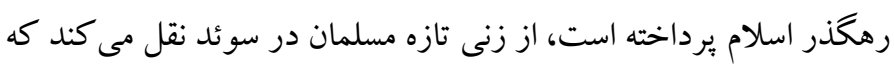

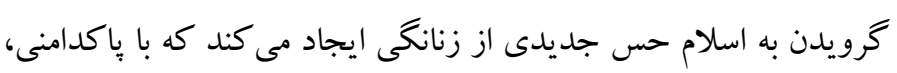

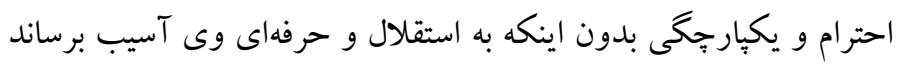
تعريف مىشود. وى مى گويد روسرى فضايى را تشكيل مىدهد كه در آن وني

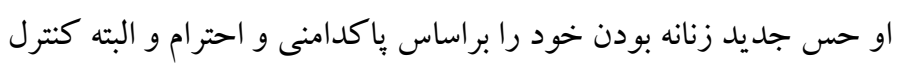

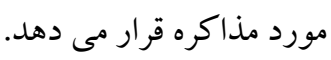

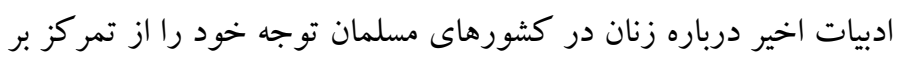
جنبههاى تاريخى و نهادى دين و مردسالارى به مفهوم سازى عامليت زنان

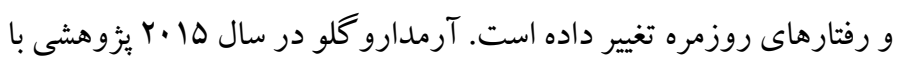

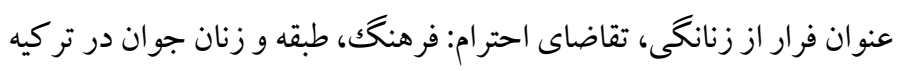

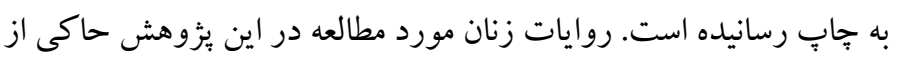

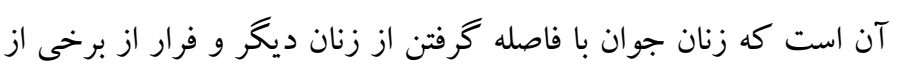
${ }^{2}$. resilience
ماساز، ورزش و ساير تمرين هاى جسمانى، ابزارهايى را براى توسعه نخرشى

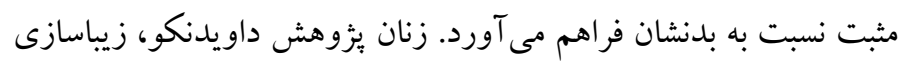

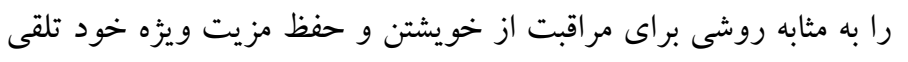

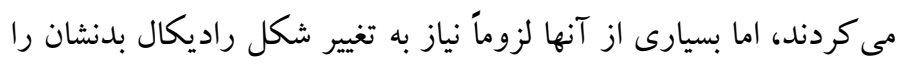

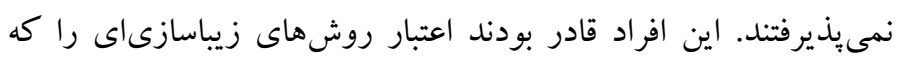

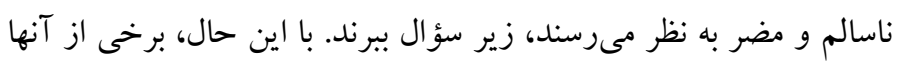

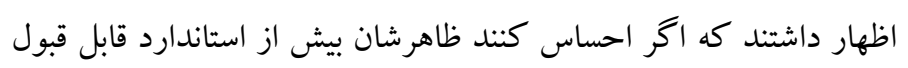

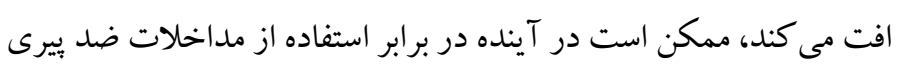

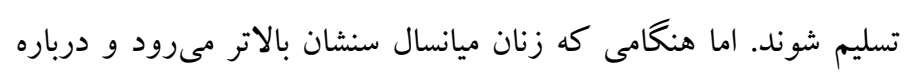

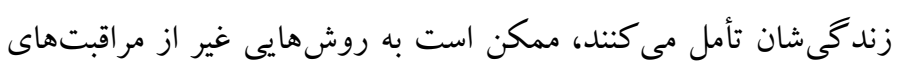

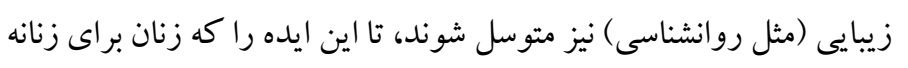

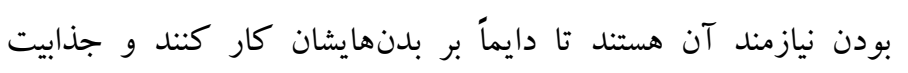
جنسى شان توسط مردان معتبر شناخته شود، زير سؤ ال ببرند.

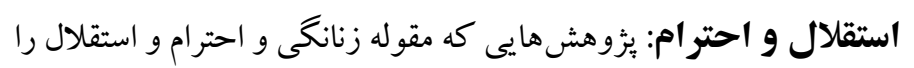
مورد بررسى قرار دادهاند، هم به مواردى اشاره دارند كه تغيير شرايط

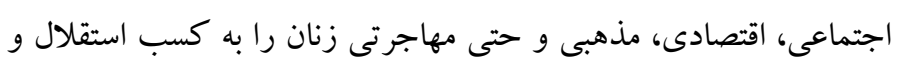

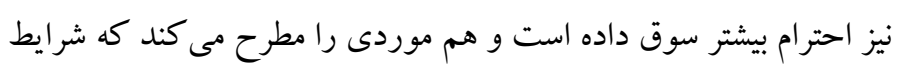

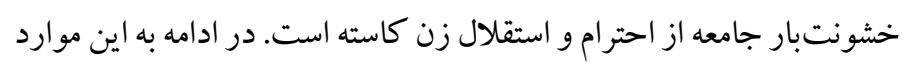
به صورت مبسوط يرداخته مى شود.

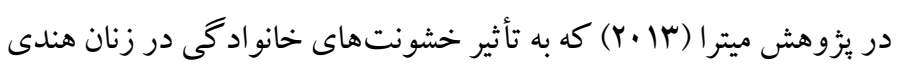
يرداخته است، مى توان شاهد از دست دادن استقلال و احترام زنان بود. از آنجا كه اين خشونت در روابط خصوصى و نزديكك تجربه مى شود، موجب

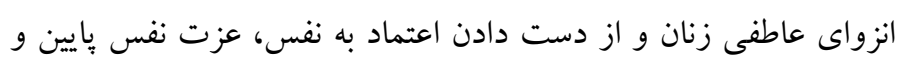

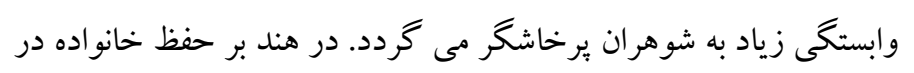

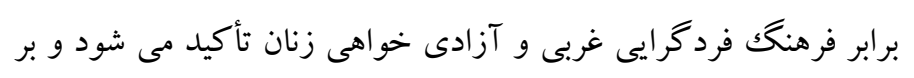

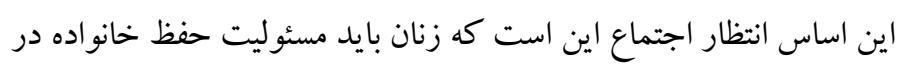
كنار هم را بر عهده بحيرند.

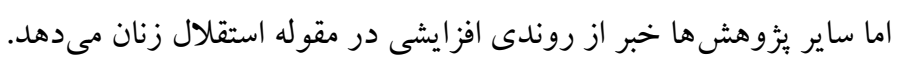

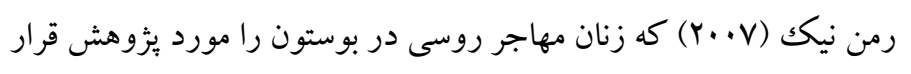

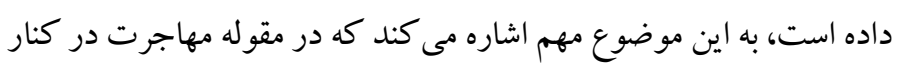

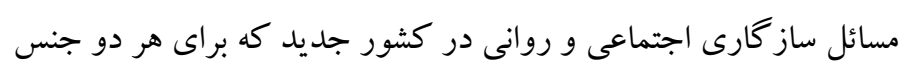

1. passivity 
تحول اخلاقى كيليكان (19Ar) بيان كرد زنان با اصولى اخلاقى هدايت

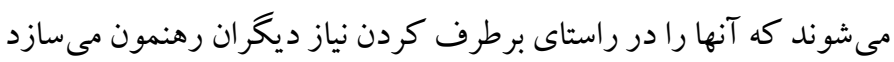
و در نتيجه بخاطر ترس از خودخواهى مانع از توجه كردن آنها به خودشان

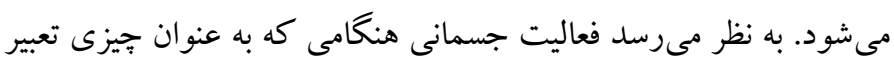

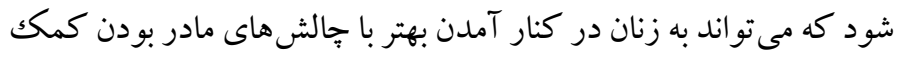
كند و در راستاى سلامت خانو اده است، معناى مثبت ترى دارد. عامل فرهنكى: در بزوهشهاى صورت گرفته در اين زمينه، مىتوان

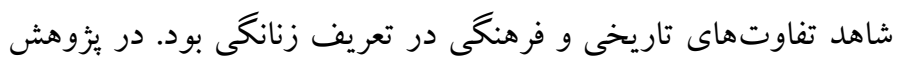
رمن نيك (Y.VV) بر روى زنان روس مهاجر كه در بخش استقلال و احترام از نتايج آن ياد شد، به تاريخجه نقش زنان در اتحاد جماهير شوروى اشاره مىشود كه در آن در دهلى •191 هم در محيط كار و هم در خانواده،

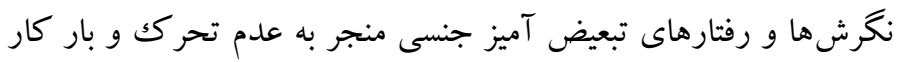
مضاعف براى اكثر زنان متاهل شد. به رغم موفقيت تحصيلى برابر يا بالاتر

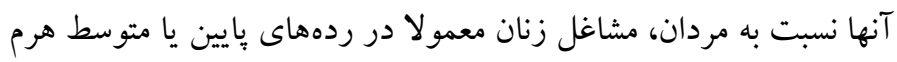
حرفها بود. با بىثباتى اقتصادى در •199 و تزلزل مشاغل مردان، بسيارى

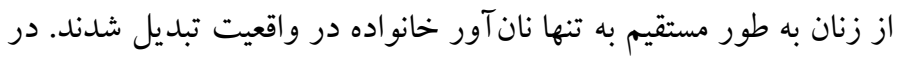
عوض، بسيارى از زنان خسته از نقشهاى متعدد، زنانكى سنتى را تثبيت كردند و آن را از طريق ظاهرى باريك و جذاب (سكسى) كه بر خرفنه از زنان خانهدار حومه شهر فيلمهاى آمريكايى و فرانسوى بود تثبيت كردند.

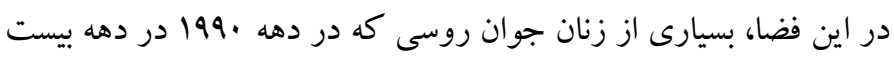

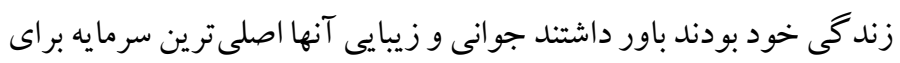

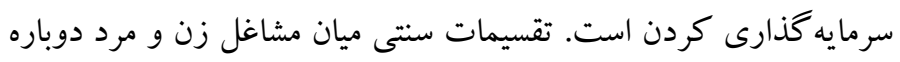
برقرار شد و زنان در حالى كه تجارت، فناورى و امور مالى را به مردان

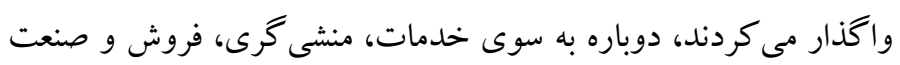
زيبايى بر گشتند.

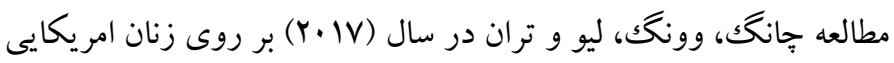

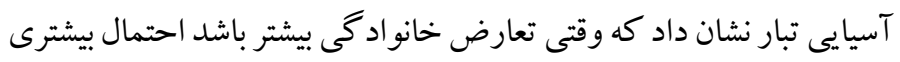
وجود دارد كه زنانكى در قالب نقشهاى مطيع توصيف شود. آنان در كار به خود به يزوهش هايى بر روى زنان كرهاى و جينى اشاره كردند كه قابل

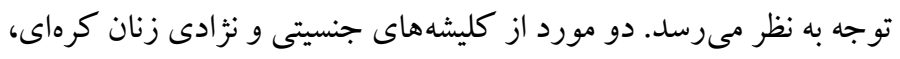

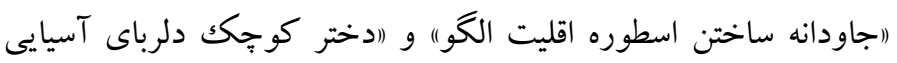

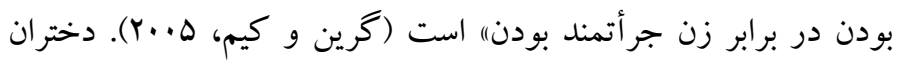

اشكال مشخص زنانگىى، زنانگى خود را بطور فعال مى سازند. آنها معمولاً

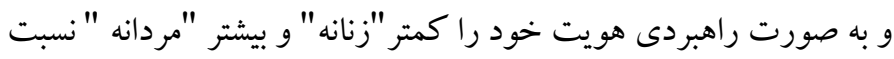

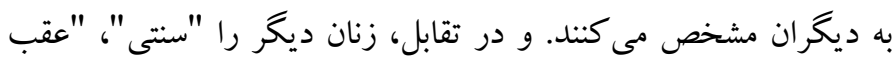

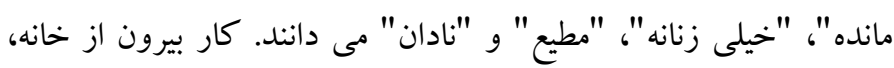

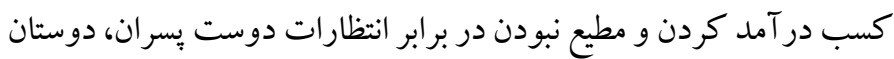

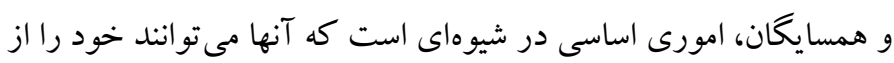

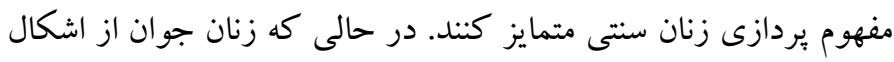

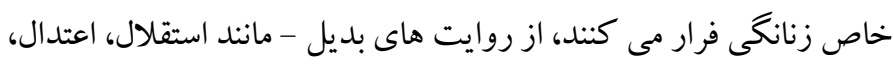
ديندارى و عفاف براى مذاكره بر سر ارزش - خود و احترام در جامعه

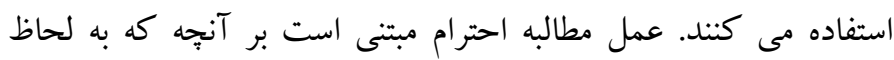
فرهنگى و اقتصادى در دسترس است.

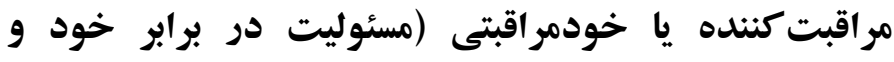

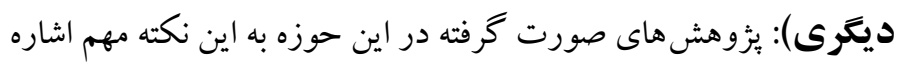

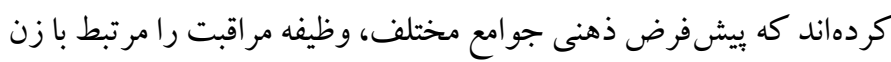

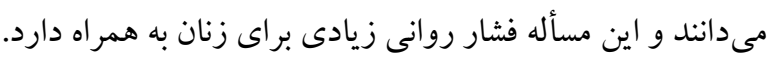

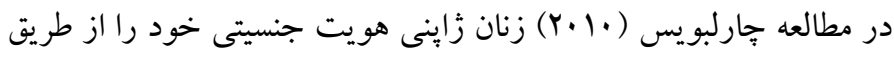
كفتمانهاى تفسيرى زنان به مثابه مراقبت كنند كان طبيعى، مادر به مثابه والد رهاب

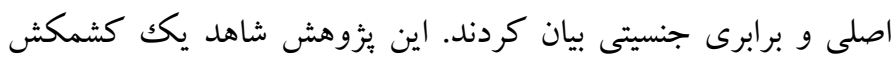

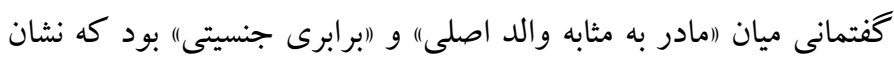
مىدهد خرد جمعى در حال ورود يكك وضعيت تغيير و فرمولبندى مجدد مئد

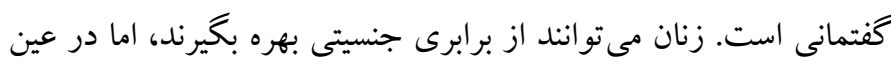

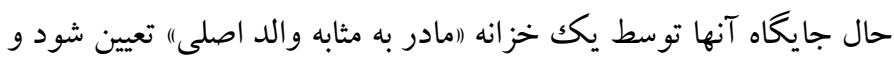

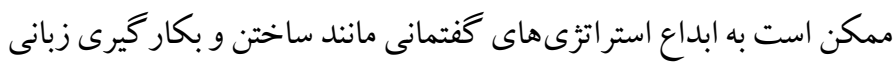

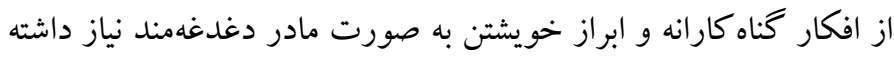

باشند، تا كشكمش ميان اين دو خزانه ظاهراً متناقض را حل كنند.

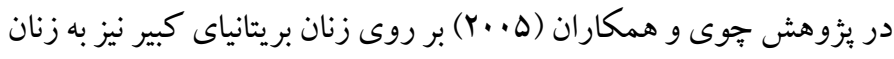
علاوه بر نقش مادرى و مراقبت از فرزند، وظايف خانهدارى و مراقبت ازي

$$
\text { ديخر ان نيز منتسب مى شود. }
$$

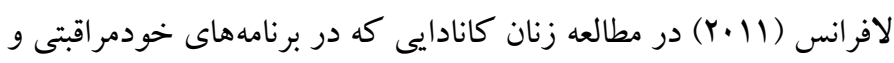
اوقات فراغت شركت مى كردند، به نوعى فرهنگك مردسالارانه غربى اشاره

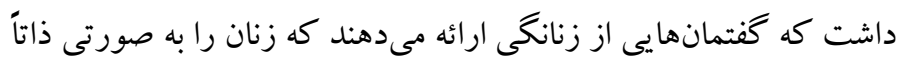

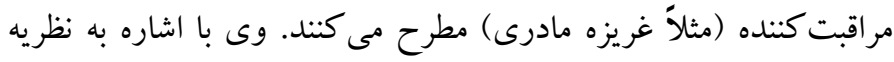


نمايد. اين يثوهش داراى محدوديتهايى بود كه از مهمترين آنها مىتوان

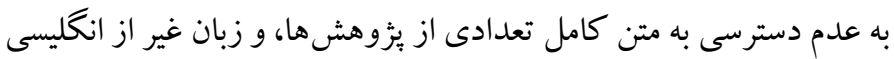

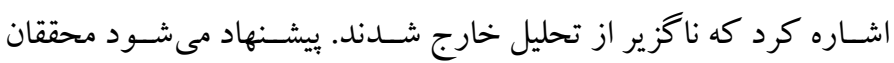

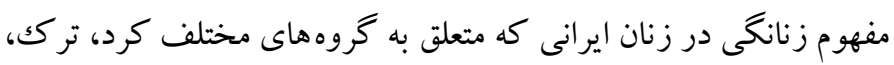

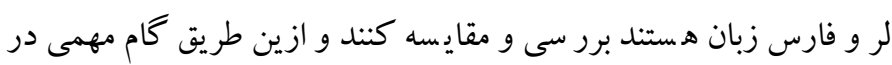
جهت مطالعه و كمكك هر جه بيشتر به زنان اين مرز و بوم بردارند.

ملاحظات اخلاقى وييروى از اصول اخلاق ئوهش: إين مقاله برگرفته از رساله دكترى نويسنده اول در

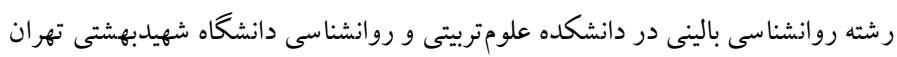

حامى مالى: اين يُروهش در قالب رساله دكترى و بدون حمايت مالى مىباشد.

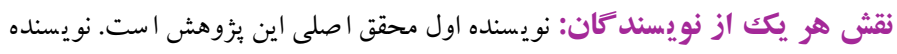

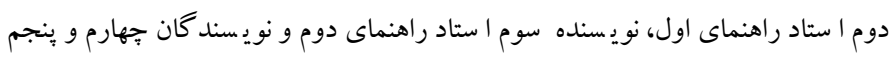

$$
\text { اساتيد مشاور رساله مىباشند. }
$$

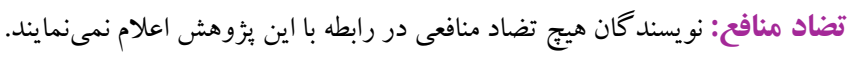

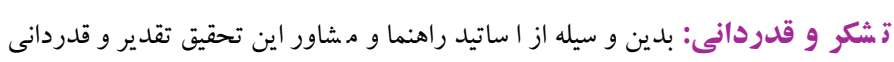

مىنمايم.

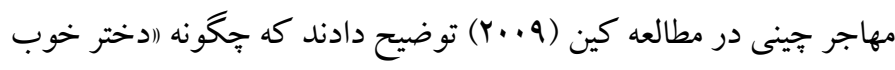

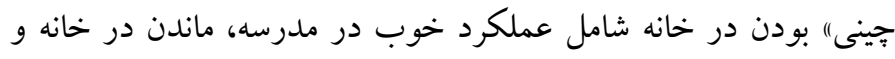
نرفتن به قرار عاشقانه بود.

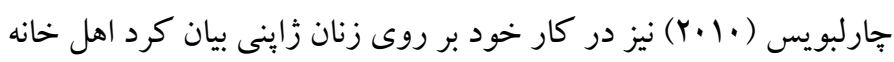

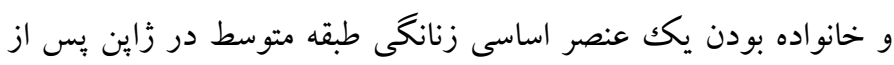

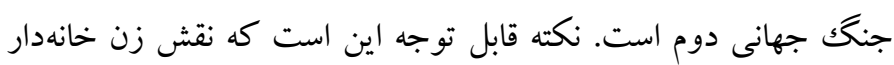

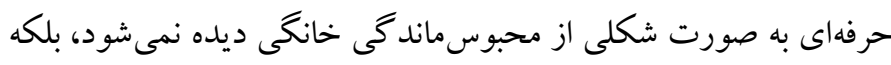
هنوز هم در ميان بسيارى از زنان محبوب است.

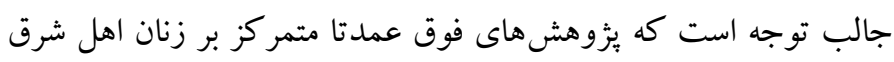

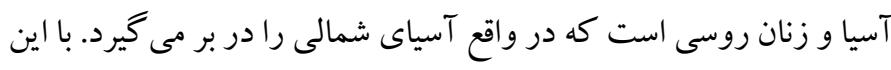
حال همجنان تفاوتهاى جشمخيرى در تعريف زنانكى در بين اين افراد

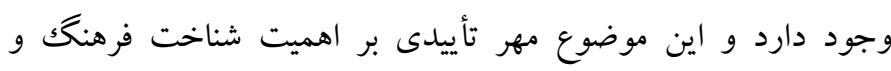
تاريخجهى يك كشور در بررسى مفهوم زنانكى است.

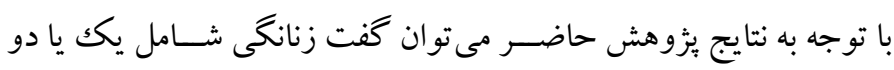

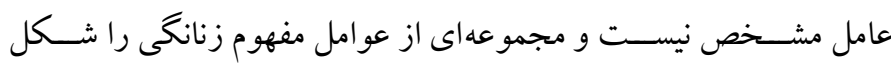

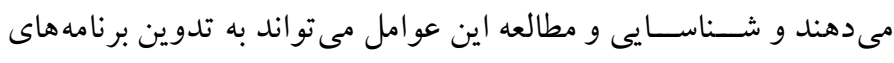

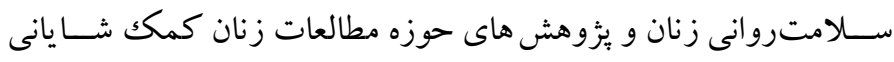




\section{References}

Alemdaroğlu, A.(2015). Escaping femininity, claiming respectability: Culture, class and young women in Turkey. Women's Studies International Forum, 53, 53-62. [Link]

Bush, J.(2000). "It's just part of being a woman": cervical screening, the body and femininity. Social Science $\&$ Medicine, 50, 429-444. [Link]

Chang, T. K., Wong, Y. J., Liu, T., Tran, K.(2017). Asian american female college students' subjective femininity conceptualizations: Using a mixedmethods approach. Journal of Multicultural Counseling and Development, 45, 260-275. [Link]

Charlebois, J.(2010). The discursive construction of femininities in the accounts of Japanese women. Discourse Studies, 12(6), 699-714. [Link]

Choi, P., Henshaw, C., Baker, S., \& Tree, J.(2005). Supermum, superwife, supereverything: performing femininity in the transition to motherhood. Journal of Reproductive and Infant Psychology, 23(2),167180. [Link]

Cole, E. R. \& Zucker, A. N.(2007). Black and white women's perspectives on femininity. Cultural Diversity and Ethnic Minority Psychology, 13(1), 19. [Link]

Davidenko, M.(2017). Searching for lost femininity: Russian middle-aged women's participation in the post-Soviet consumer culture. Journal of Consumer Culture. [Link]

Fiaveha, D. Y., Lzugbarab, Ch. O., Okyerefoa, M. P. K., Reysooc, F., \& Fayorseya, C. K.(2014). Constructions of masculinity and femininity and sexual risk negotiation practices among women in urban Ghana. Culture, Health \& Sexuality: An International Journal for Research, Intervention and Care, 17, 650-662. [Link]

Fritsch, E., ellman, P., basseches, H., Elmendorf, S., goodman, N., helm, F., Rockwell, SH.(2001) The riddle of femininity: the interplay of primary femininity and the castration complex in analytic listening. Int. J. Psychoanal, 82, 1171-1183. [Link]

Guillen Fabi, S., \& Willett, M.(2019). Youth versus femininity: What are we truly trying to achieve?. Journal of Cosmetic Dermatology, 00, 1-3.[Link]

Hamzavi-Abedi F, Bagherian F, Mazaheri M.(2015). Culture, immigration and femininity perception: Comparing young Iranian, Canadian, and IranianCanadian immigrant women.Journal of psychological science,(52) 13, 530-541.(Persian) [Link]
Han, Ch., Zhang, Y., Lei, X., Li, X., Morrison, E. R., \& $\mathrm{Wu}, \quad$ Y.(2020). Single dose testosterone administration increases men's facial femininity preference in a Chinese population. Psychneuroendocrinology [Link]

Hardy, T. L. D., Boliek, C. A., Wells, K., Deraden, C., Zalmanowitz, C., \& Rieger, J. M. (2016). Pretreatment acoustic predictors of gender, femininity, and naturalness ratings in individuals with male-to-female gender identity. American Journal of Speech-Language Pathology, 25, 125137.[Link]

Kachel, S., Steffens, M. C., \& Niedlich, C.(2016). Traditional masculinity and femininity:validation of a new scale assessing gender roles. Frontiers in Psychology, 7, 956.[Link]

Karupiah, P.(2016). Marital status and the influence of emphasized femininity on the romantic relationships of tamil single mothers in malasia. Contemporary Perspectives in Family Research, 10, 375-393. [Link]

Kelly, M., Inoue, K., Barratt, A., Bateson, D., Rutherford, A. \& Ritchers, J.(2016). Performing (heterosexual) femininity: female agency and role in sexual life and contraceptive use - a qualitative study in Australia. Culture, Health \& Sexuality, 19(2):240-255 [Link]

Khodabande loo Y, Pour Hosein R, Gholamali Lavasani M, MotamedYeganeh N.(2016). Investigating the effectiveness of self-review cognitive method on body images of the female students of university of Tehran. Journal of psychological science,(55) 14, 301-320. (Persian) [Link]

Komatsu, H., Yagasaki, K., Shoda, R., Chung, Y., Iwata, T., Sugiyama, J., Fujii, T.(2014). Repair of the threatened feminine identity, experience of women with cervical cancer undergoing fertility preservation surgery. Cancer Nursing, 37(1), 75-82. [Link]

Lafrance, M. N.(2011). Reproducing, resisting and transcending discourses of femininity: a discourse analysis of women's accounts of leisure. Qualitative Research in Sport, Exercise and Health, 3(1), 8098.[Link]

Leavy, P., Gnog, A., \& Sardi ross, L.(2009). Femininity, masculinity, and body image issues among collegesge women: an in-depth and written interview study of the mind-body dichotomy. The Qualitative Report, 14(2), 261-292. [Link]

Mahalik, J., Morray, E., Coonerty-Femiano, A., Ludlow, L., Slattery, S., and Smiler, A.(2005). Development 
of conformity to feminine norms. Sex roles, 52(7/8), 417-435. [Link]

Marshall, K., Chamberlain, K., \& Hodgetts, D.(2018). Female bodybuilders on Instagram: Negotiating an empowered femininity.Feminism \& Psychology, 0(0), 1-24.[Link]

McGinty, A. M.(2007). Formation of alternative femininities through Islam: Feminist approaches among muslim converts in Sweden. Women's Studies International Forum, 30, 474-485.[Link]

Mitra, N.(2013). Intimate violence, family, and femininity: Women's narratives on their construction of violence and self. Violence Against Women. [Link]

Moreno-Bella, E., Willis, G. B. \& Moya, M.(2019). Economic inequality and masculinity-femininity: the prevailing perceived traits in higher unequal contexts are masculine. Frontiers in Psychology, 10, 1590.[Link]

Nicholls, E.(2019). 'I feel like I have to become part of that identity': Negotiating femininities and friendships through alcohol consumption in Newcastle, UK. International Journal of Drug Policy. [Link]

Noblit GW, Hare RD. Meta-ethnography: Synthesizing qualitative studies. Newbury Park, CA: Sage; 1988. [Link]

Pereira, K. J., Varella, M. A. C., Kleisner, K., Pavlovic, O., Valentova, J. V.(2019). Positive association between facial and vocal femininity/masculinity in women but not in men. Behavioural Processes, 164, 25-29. [Link]

Quach, T.(2008). Femininity and sexual agency among young unmarried women in Hanoi. Culture, Health \& Sexuality, 10, S151-S161.[Link]

Reischer, E., Koo, K. S.(2004). The Body Beautiful: Symbolism and agency in the social world. Annual Review of Anthropology, 33, 297-317. [Link]

Remennick, L.(2007). "Being a woman is different here": Changing perceptions of femininity and gender relations among former Soviet women living in Greater Boston. Women's Studies International Forum, 30, 326-341.[Link]

Rutagumirwa, S. K., Bailey, A.(2017). "I have to listen to this old body": Femininity and the aging body. Gerontologist, 0(0) , 1-10.[Link]

Salazar, M., Goicolea, I. \& Öhman, A.(2016). Respectable, disreputable, or rightful? young nicaraguan women's discourses on femininity, intimate partner violence, and sexual abuse: a grounded theory situational analysis. Journal of
Aggression, Maltreatment \& Trauma, 25(3), 315332.[Link]

Shea, M., Wong, Y., Wang, S., Sherry Wang, S., Jimenez, V., Hickman, S., \& LaFollette, J.(2014). Toward a constructionist perspective of examining femininity experience: the development and psychometric properties of the subjective femininity stress scale. Psychology of Women Quarterly. 38(2), 275291.[Link]

Sheikh M, Moslemi Nejad M.(2016). Comparison of body image anxiety of athletic and nonathletic elder women: The mediator role of body index with relation to self-respect and body image anxiety. Journal of psychological science,(57)15, 98-112. (Persian) [link]

Stromback, M., Formark, B., Wiklund, M. \& MalmgrenOlsson, E.(2014). The corporeality of living stressful femininity: a gender-theoretical analysis of young swedish women's stress experiences. Young. 22(3), 271-289. [Link]

Zajdow, G. (2007). "The Gender Order." In Public Sociology: An Introduction to Australian Society. Sydney: Allen \& Unwin. [Link]

Zinn, M.B., Hondagneu-Sotelo, P., and Messner, M.A.(2011). Gender through the prism of difference. New York: Oxford University Press. [Link] 